\title{
Aplicación de ecuaciones de estado cúbicas y desarrollo de correlaciones numéricas para el cálculo de propiedades termodinámicas en saturación de refrigerantes para su uso en microcontroladores
}

\author{
García del Valle J. \\ Área de Energía, Facultad de Ingeniería Civil y Mecánica, Universidad Técnica de Ambato, Ambato, Ecuador. \\ Autor para correspondencia: jav.garcia@uta.edu.ec \\ Fecha de recepción: 4 de octubre 2017 - Fecha de aceptación: 21 de octubre 2018
}

\begin{abstract}
RESUMEN
Actualmente las librerías para cálculo de propiedades termodinámicas se basan en la ecuación de la energía de Helmholtz, cuya implementación informática sobrepasa los requerimientos de memoria de la mayoría de microcontroladores, estando además escritas en lenguajes de alto nivel. En la presente investigación, después de estudiar el comportamiento de cinco variantes de la ecuación de Peng-Robinson, se propuso el desarrollo de ecuaciones sencillas para las propiedades de saturación que igualasen o mejorasen las predicciones anteriores, al mismo tiempo que fuesen de fácil implementación en lenguaje $\mathrm{C}$, para su uso en microcontroladores. Partiendo de las propiedades obtenidas por la librería de referencia RefProp, se realizarán ajustes multiparamétricos, por mínimos cuadrados, de distintas propiedades termodinámicas, en base a un número de ecuaciones propuestas. Tanto una discusión de la mejor función para cada propiedad, así como los coeficientes e incertidumbres medias y locales han sido reportados para un número de refrigerantes comunes.
\end{abstract}

$\underline{\text { Palabras clave: }}$ Refrigerante, microcontrolador, saturación, propiedad termodinámica, lenguaje C.

\begin{abstract}
The state-of-the-art of computer libraries in the field of thermodynamic properties are based on the Helmholtz energy equation. Unfortunately, the use of high-level programming languages and its complex implementation often exceeds the memory limit of most microcontrollers. In this context, after examination of the behavior of five variants of the Peng-Robinson equation, a set of simple equations for the saturation properties of common refrigerants that exceeded the precision of the former ones were developed. The proposed correlations are directly programmable in $\mathrm{C}$ language, facilitating the implementation in microcontrollers. Several prototype equations were fitted to the thermodynamic data in the reference RefProp library for a few thermodynamics properties by means of a non-linear multiparametric least-squares fitting procedure. Finally, a discussion of the best function for each property, as well as the average and local coefficients and uncertainties have been reported for several common refrigerants.
\end{abstract}

Keywords: Refrigerant, microcontroller, saturation, thermodynamic property, $\mathrm{C}$ language.

\section{INTRODUCCIÓN}

El uso de microcontroladores para el control térmico en electrodomésticos y pequeña maquinaria industrial, relacionada con aplicaciones frigoríficas, es cada día más común. Desde válvulas de expansión electrónicas para ciclos frigoríficos (CAREL Industries S.p.A., 2017) hasta analizadores y cargadores de gas (YellowJacket, 2017), su funcionamiento pasa por el uso de un microcontrolador. En estos casos, el uso de propiedades termodinámicas es necesario para relacionar la presión con la temperatura para un refrigerante en saturación. Otros parámetros como entalpías y calores específicos pueden ser también de utilidad. Los controladores comerciales implementan código cerrado, el cual no está disponible para su implementación en plataformas abiertas de desarrollo, tipo Arduino y sus derivados. Dado además que existen restricciones en cuanto a memoria en las citadas plataformas, la implementación directa de librerías de referencia para cálculos termodinámicos como RefProp (NIST, 2017) o el proyecto de código abierto CoolProp
(Bell, Wronski, Quoilin \& Lemort, 2014), se convierten en alternativas inviables.

El estado del arte en la determinación de propiedades termodinámicas se basa en la proposición y posterior ajuste de ecuaciones multiparamétricas para la energía de Helmholtz en función de la temperatura y densidad del fluido (Span, 2000). A partir de dicha expresión y mediante termodinámica diferencial, el resto de propiedades termodinámicas pueden ser calculadas. A pesar del consenso y uso extensivo de funciones de estado basadas en la energía de Helmholtz (Wolfgang \& Kretzschmar, 2008), ecuaciones de estado no analíticas basadas en la ecuación de Benedict-Web-Rubbin o Martin-Hou son todavía empleadas en literatura técnica (DuPont TM Suva $\left.{ }^{\circledR}, 2017\right)$. Formulaciones más simples, basadas en la ecuación de Peng-Robinson (Poling, Prausnitz \& O'Connell, 2001), están en desuso excepto en aplicaciones especializadas (Brown, 2007). Para las ecuaciones de estado anteriores, independientemente de su morfología, aparecen formulaciones implícitas que requieren soluciones numéricas de ecuaciones no lineales. Dichas 
soluciones no son sólo exigentes para un microcontrolador, sino que además se hace necesario la programación de algoritmos robustos con manejo de errores y excepciones automáticas. Además, la precisión simple en operaciones de coma flotante encontradas en microcontroladores de 8 bit pueden introducir errores máquina apreciables en soluciones iterativas de ecuaciones.

Una solución simple a este problema es la construcción de una base de datos estructurada e interpolación para la propiedad termodinámica deseada. Tanto interpolaciones lineales como métodos mejorados de interpolación, por ejemplo, splines cúbicas, han sido propuestos en la literatura (Atalay \& Coban, 2015) con altos grados de representatividad en relación con las propiedades exactas. Esta aproximación al problema se traduce en rutinas rápidas y robustas de cálculo con la contrapartida de una base de datos que puede ser de tamaño moderado en función de la región y el número de propiedades termodinámicas que se quieran cubrir. Si, además, como es el caso en la presente investigación, se quiere abarcar un número extenso de refrigerantes, se puede incurrir de nuevo en restricciones de memoria flash del microcontrolador. Otro método propuesto en la literatura se basa en la construcción de un sistema de ecuaciones implícitas para una cierta región con una formulación más simple que la ecuación original de Helmholtz (Zhao, Ding \& Wu, 2009). Aunque el método produce buenos resultados, se tienen de nuevo los problemas mencionados con anterioridad en relación con los esquemas numéricos para resolver sistemas no lineales. Para microcontroladores sería preferible una formulación explícita del problema. Expresiones polinómicas han sido propuestas con anterioridad (Cleland, 1994; Vikrant \& Radermacher, 2014), sin embargo, un número reducido de propiedades o refrigerantes han sido cubiertos en dichos estudios. Ecuaciones más complejas, basadas en funciones exponenciales y/o logarítmicas, también ha sido sugeridas para la presión de saturación (Iglesias-Silva, Miller, Ceballos, Hall \& Holste, 1995) o para describir distintas propiedades de un único refrigerante (Affandia, Mamat, Kanafiah \& Khalid, 2013).

En el presente estudio, tras analizar el comportamiento de ecuaciones de estado cúbicas tipo Peng-Robinson, se persigue obtener formulaciones explícitas óptimas y con error conocido para 14 refrigerantes comunes y para 9 propiedades termodinámicas en saturación como: presión, densidad, energía interna, entalpía, entropía, calor específico a presión constante y a volumen constante, velocidad del sonido y coeficiente de Joule-Thompson en función de la temperatura de saturación.

\section{MÉTODOS}

\subsection{Estudio de ecuaciones cúbicas tipo Peng- Robinson}

En una primera etapa se ha estudiado el empleo de ecuaciones de estado cúbicas para obtener las condiciones de equilibrio líquido-vapor. En particular se ha optado por la ecuación de Peng-Robinson. Si bien para el cálculo de entalpías y entropías es necesario una expresión para la entalpía de gas ideal, el equilibrio líquido-vapor puede ser calculado únicamente mediante la ecuación de estado, si bien dicho procedimiento no es directo. Para cada isoterma, aparece la resolución de un sistema no lineal de tres ecuaciones que involucra la integral de Maxwell, ecuación (1), y la ecuación de estado para líquido y vapor. Como resultado se obtiene la presión, densidad de líquido y densidad de vapor. Para la resolución de dicho sistema se ha realizado un programa en $\mathrm{C}++$ que, de forma genérica, resuelve el equilibrio para cualquier función cúbica de estado (García, 2018). Dado que las curvas son suaves, se ha empleado una integral por la regla del trapecio y el método de la secante ha sido empleado para la resolución del sistema.

$$
p\left(v_{v}-v_{l}\right)=\int_{v_{v}}^{v_{l}} p d v
$$

La ecuación de estado de Peng-Robinson ha sido ampliamente estudiada en la literatura. En LopezEcheverry, Reif-Acerman \& Araujo-Lopez (2017) se hace una revisión exhaustiva de las diferentes modificaciones propuestas por diferentes autores para mejorar la predicción de la formulación original (Peng \& Robinson, 1979). Dos caminos han sido propuestos. El primero, siguiendo la idea original, propone obtener los coeficientes de la ecuación de estado en función de parámetros físicos del fluido, tales como el factor acéntrico, factor de compresibilidad en el punto crítico y temperatura reducida, fundamentalmente. El segundo método propone una formulación específica para cada fluido, basada en el ajuste de diferentes coeficientes dentro de la ecuación de estado. Mientras que el primer procedimiento tiene una aplicación general, el segundo es aplicable únicamente a una sustancia en particular. Entre las formulaciones que adoptan el primer criterio, se han considerado para la presente investigación, además de la formulación original, las propuestas por Peng \& Robinson (1980), Perez (1987), Hewitt, McMullan, Evans \& Mongoney (1996) y Figueira, Lugo \& Olivera-Fuentes (2007).

Tabla 1. Refrigerantes estudiados junto con el rango de temperatura considerado.

\begin{tabular}{cccccccc}
\hline & Refrigerante & $\mathrm{T} \mathrm{min}\left[{ }^{\circ} \mathrm{C}\right]$ & $\mathrm{T} \max \left[{ }^{\circ} \mathrm{C}\right]$ & & Refrigerante & $\mathrm{T} \min \left[{ }^{\circ} \mathrm{C}\right]$ & $\mathrm{T} \max \left[{ }^{\circ} \mathrm{C}\right]$ \\
\hline 1 & R717 & -60 & 80 & 8 & $\mathrm{R} 22$ & -60 & 80 \\
2 & R600 & -60 & 80 & 9 & $\mathrm{R} 32$ & -60 & 74.05 \\
3 & R744 & -56.15 & 26.95 & 10 & $\mathrm{R} 404 \mathrm{a}$ & -60 & 68.05 \\
4 & R600a & -60 & 80 & 11 & $\mathrm{R} 407 \mathrm{c}$ & -60 & 80 \\
5 & R1234yf & -53.15 & 80 & 12 & $\mathrm{R} 410 \mathrm{a}$ & -60 & 67.25 \\
6 & R12 & -60 & 80 & 13 & $\mathrm{R} 507 \mathrm{a}$ & -60 & 66.45 \\
7 & R134a & -60 & 80 & 14 & $\mathrm{R} 718$ & 0.85 & 80 \\
\hline
\end{tabular}




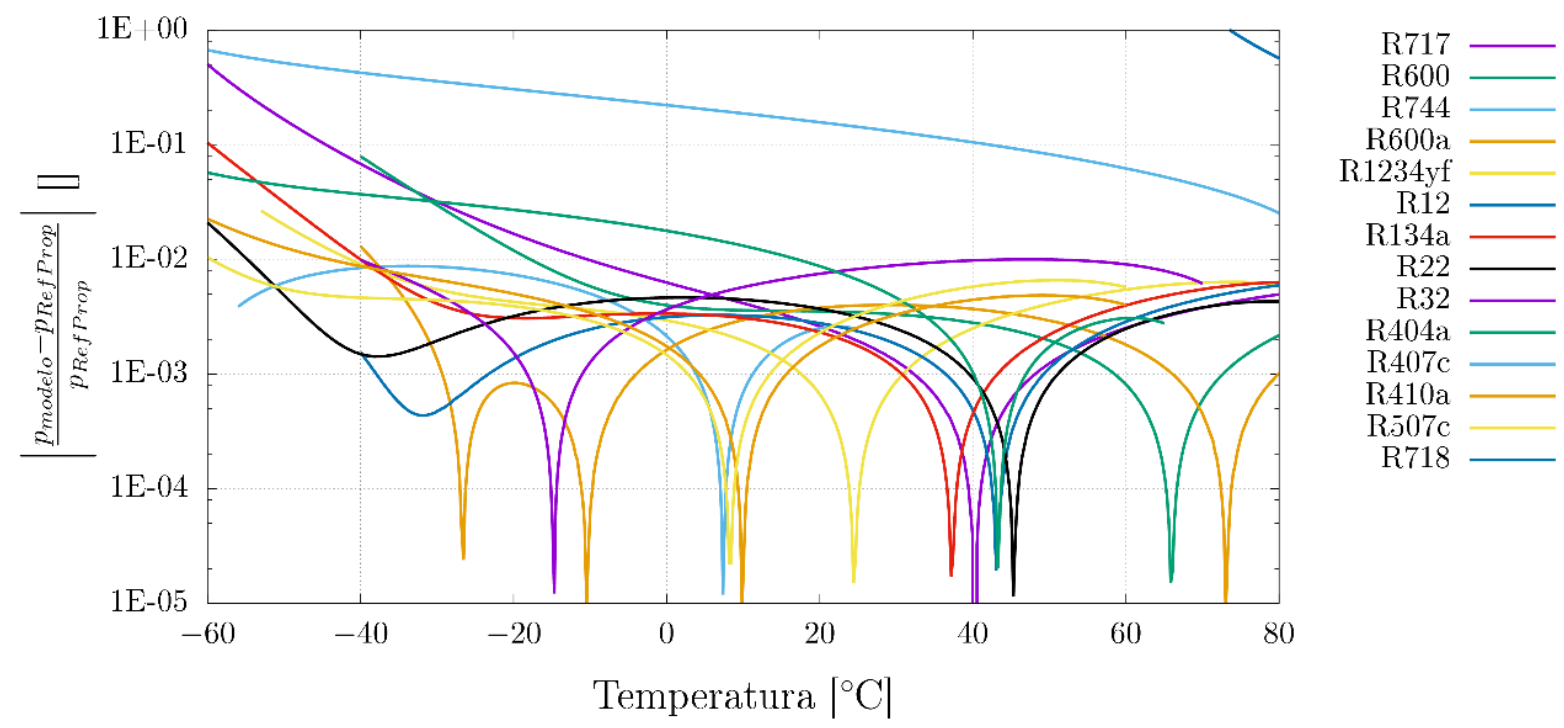

Figura 1 Error relativo absoluto para la presión calculada por la ecuación de Peng-Robinson para los distintos fluidos considerados.

Los fluidos considerados y el rango de trabajo quedan detallados en la Tabla 1. Se ha considerado desde el máximo entre el punto triple y $-60^{\circ} \mathrm{C}$ hasta el mínimo entre el punto crítico y $80^{\circ} \mathrm{C}$, tal y como se muestra en la Tabla 1. Se han considerados fluidos y rangos de trabajos empleados comúnmente en refrigeración y climatización.

Para el estudio de la ecuación de Peng-Robinson, se han considerado 500 isotermas entre el valor máximo y el mínimo mostrado para cada fluido, calculándose, para cada una de ellas, las presiones de equilibrio y densidades de líquido y vapor. Se muestra en la Figura 1 el error relativo para los diferentes refrigerantes y temperaturas considerados para la predicción de la presión. Dado que se ha tomado el valor absoluto, los mínimos indican un paso por cero.
Para cuantificar numéricamente el error se ha incluido la Tabla 2. En la misma se detalla el error relativo promedio de todas las isotermas para la presión en función de los diferentes refrigerantes y correlaciones estudiadas.

Además, se ha incluido, en la parte baja de la tabla, el error promedio para todos los refrigerantes y las propiedades de presión, densidad de líquido y vapor. El error relativo promedio se calcula de acuerdo la ecuación (2). El error mínimo para cada propiedad ha sido resaltado en la Tabla 2. Puede verse que las nuevas formulaciones no mejoran la predicción original de Peng-Robinson, a excepción de la propuesta por Pérez Casas (1987) que mejora ligeramente la predicción para la densidad de líquido.

$$
\text { Error }=\frac{1}{n} \Sigma\left|\frac{x_{\text {ajuste }}-x_{\text {RefProp }}}{x_{\text {RefProp }}}\right|
$$

Tabla 2. Promedio del error relativo para la presión de saturación para los refrigerantes considerados en función de cinco correlaciones tipo Peng-Robinson. Se ha incluido el error total medio para todos los refrigerantes y las propiedades de presión de saturación, densidad de líquido y densidad de vapor.

\begin{tabular}{lccccc}
\hline \multicolumn{1}{c}{ Refrigerant } & $\begin{array}{c}\text { (Peng \& } \\
\text { Robinson, 1976) }\end{array}$ & $\begin{array}{c}\text { (Peng \& } \\
\text { Robinson, 1980) }\end{array}$ & $\begin{array}{c}\text { (Perez, 1987) } \\
\text { (Hewitt et al., } \\
1996)\end{array}$ & $\begin{array}{c}\text { (Figueira et al., } \\
\text { 2007) }\end{array}$ \\
\hline R717 & $3.10 \mathrm{E}-02$ & $2.31 \mathrm{E}-01$ & $3.87 \mathrm{E}-02$ & $2.03 \mathrm{E}+00$ & $5.77 \mathrm{E}-02$ \\
R600 & $8.28 \mathrm{E}-03$ & $3.17 \mathrm{E}-01$ & $1.40 \mathrm{E}-02$ & $1.42 \mathrm{E}+00$ & $1.88 \mathrm{E}-01$ \\
R744 & $5.03 \mathrm{E}-03$ & $1.40 \mathrm{E}-01$ & $7.28 \mathrm{E}-03$ & $3.25 \mathrm{E}-01$ & $3.96 \mathrm{E}-02$ \\
R600a & $2.39 \mathrm{E}-03$ & $3.03 \mathrm{E}-01$ & $6.83 \mathrm{E}-03$ & $9.88 \mathrm{E}-01$ & $1.89 \mathrm{E}-01$ \\
R1234yf & $4.93 \mathrm{E}-03$ & $1.21 \mathrm{E}-01$ & $9.88 \mathrm{E}-03$ & $9.76 \mathrm{E}-01$ & $2.87 \mathrm{E}-02$ \\
R12 & $2.30 \mathrm{E}-03$ & $2.58 \mathrm{E}-01$ & $5.15 \mathrm{E}-03$ & $6.63 \mathrm{E}-01$ & $1.52 \mathrm{E}-01$ \\
R134a & $8.24 \mathrm{E}-03$ & $4.82 \mathrm{E}-02$ & $1.81 \mathrm{E}-02$ & $1.62 \mathrm{E}+00$ & $1.50 \mathrm{E}-01$ \\
R22 & $3.51 \mathrm{E}-03$ & $2.07 \mathrm{E}-01$ & $7.64 \mathrm{E}-03$ & $9.06 \mathrm{E}-01$ & $7.83 \mathrm{E}-02$ \\
R32 & $6.40 \mathrm{E}-03$ & $9.76 \mathrm{E}-02$ & $7.18 \mathrm{E}-03$ & $5.87 \mathrm{E}-01$ & $6.94 \mathrm{E}-02$ \\
R404a & $1.85 \mathrm{E}-02$ & $1.06 \mathrm{E}-01$ & $2.45 \mathrm{E}-02$ & $8.20 \mathrm{E}-01$ & $4.06 \mathrm{E}-02$ \\
R407c & $1.72 \mathrm{E}-01$ & $1.66 \mathrm{E}-01$ & $1.81 \mathrm{E}-01$ & $8.36 \mathrm{E}-01$ & $6.95 \mathrm{E}-02$ \\
R410a & $5.39 \mathrm{E}-03$ & $8.98 \mathrm{E}-02$ & $1.07 \mathrm{E}-02$ & $8.86 \mathrm{E}-01$ & $5.07 \mathrm{E}-02$ \\
R507a & $4.13 \mathrm{E}-03$ & $1.01 \mathrm{E}-01$ & $8.84 \mathrm{E}-03$ & $8.44 \mathrm{E}-01$ & $5.29 \mathrm{E}-02$ \\
R718 & $4.70 \mathrm{E}-01$ & $3.80 \mathrm{E}-01$ & $5.10 \mathrm{E}-01$ & $1.14 \mathrm{E}+01$ & $2.40 \mathrm{E}-01$ \\
\hline Total p & $5.30 \mathrm{E}-02$ & $1.83 \mathrm{E}-01$ & $6.07 \mathrm{E}-02$ & $1.73 \mathrm{E}+00$ & $1.00 \mathrm{E}-01$ \\
Total $\rho$ liquido & $6.21 \mathrm{E}-02$ & $6.23 \mathrm{E}-02$ & $6.19 \mathrm{E}-02$ & $1.05 \mathrm{E}-01$ & $7.06 \mathrm{E}-02$ \\
Total $\rho$ vapor & $5.17 \mathrm{E}-02$ & $1.97 \mathrm{E}-01$ & $6.04 \mathrm{E}-02$ & $1.90 \mathrm{E}+00$ & $1.06 \mathrm{E}-01$ \\
\hline
\end{tabular}




\subsection{Proposición de expresiones simples y método de ajuste}

Una vez estudiado el comportamiento de la ecuación de estado de Peng-Robinson y las variantes consideradas, el propósito de esta sección es proponer correlaciones simples para las propiedades estudiadas con anterioridad, entre otras. La comparación del error entre la ecuación de Peng-Robinson y las correlaciones propuestas dará una magnitud de la validez de éstas. La obtención de propiedades termodinámicas de referencia se ha realizado empleando la base de datos termodinámicos RefProp, del National Institute of Standards (NIST, 2017), disponible en línea. La base de datos RefProp se basa en las funciones de Helmholtz más precisas desarrolladas hasta la fecha para cada refrigerante, pudiéndose considerar un error despreciable para el caso del cálculo de propiedades de saturación. Se consideró intervalos de $0.1^{\circ} \mathrm{C}$ para la obtención de datos de las diferentes isotermas.

Las propiedades termodinámicas consideradas para las correlaciones simples, junto con sus respectivas unidades, se muestran en la Tabla 3. Dado que hay que considerar las propiedades tanto para la fase líquido como para vapor, se tiene un total de 18 propiedades a evaluar. En el caso de la presión, hay que considerar que, si bien para fluidos puros y mezclas azeotrópicas el valor de presión para líquido como para vapor es el mismo, en el caso de fluidos zeotrópicos (R404a, R407c y R410a) su valor difiere, de ahí su consideración en la Tabla 3.

Tabla 3. Detalle de propiedades termodinámicas junto con las unidades consideradas.

\begin{tabular}{|c|c|c|}
\hline \multicolumn{3}{|c|}{ Propiedad } \\
\hline 1 & plíquido & {$[\mathrm{kPa}]$} \\
\hline 2 & $\mathrm{p}_{\text {vapor }}$ & {$[\mathrm{kPa}]$} \\
\hline 3 & $\rho_{\text {líquido }}$ & {$\left[\mathrm{Kg} \mathrm{m}^{-3}\right]$} \\
\hline 4 & $\rho_{\text {vapor }}$ & {$\left[\mathrm{Kg} \mathrm{m}^{-3}\right]$} \\
\hline 5 & ulíquido & {$\left[\mathrm{J} \mathrm{kg}^{-1}\right]$} \\
\hline 6 & $\mathrm{~h}_{\text {líquido }}$ & {$\left[\mathrm{J} \mathrm{kg}^{-1}\right]$} \\
\hline 7 & Slíquido & {$\left[\mathrm{J} \mathrm{Kg}^{-1} \mathrm{~K}^{-1}\right]$} \\
\hline 8 & cVlíquido & {$\left[\mathrm{J} \mathrm{Kg}^{-1} \mathrm{~K}^{-1}\right]$} \\
\hline 9 & cplíquido & {$\left[\mathrm{J} \mathrm{Kg}^{-1} \mathrm{~K}^{-1}\right]$} \\
\hline 10 & $\mathrm{SS}_{\text {líquido }}$ & {$\left[\mathrm{m} \mathrm{s}^{-1}\right]$} \\
\hline 11 & $\mu_{\mathrm{JT}, \text { líquido }}$ & {$\left[\mathrm{K} \mathrm{Pa}^{-1}\right]$} \\
\hline 12 & $\mathrm{U}_{\text {vapor }}$ & {$\left[\mathrm{J} \mathrm{kg}^{-1}\right]$} \\
\hline 13 & hvapor & {$\left[\mathrm{J} \mathrm{kg}^{-1}\right]$} \\
\hline 14 & Svapor & {$\left[\mathrm{J} \mathrm{Kg}^{-1} \mathrm{~K}^{-1}\right]$} \\
\hline 15 & CVvapor & {$\left[\mathrm{J} \mathrm{Kg}^{-1} \mathrm{~K}^{-1}\right]$} \\
\hline 16 & $\mathrm{cp}_{\text {vapor }}$ & {$\left[\mathrm{J} \mathrm{Kg}^{-1} \mathrm{~K}^{-1}\right]$} \\
\hline 17 & $\mathrm{SS}_{\text {vapor }}$ & {$\left[\mathrm{m} \mathrm{s}^{-1}\right]$} \\
\hline 18 & $\mu \mathrm{JT}$,vapor & {$\left[\mathrm{K} \mathrm{Pa}^{-1}\right]$} \\
\hline
\end{tabular}

Se han propuesto un total de 13 prototipos de funciones para el ajuste de las propiedades termodinámicas consideradas, según se muestra en la Tabla 4, que engloban expresiones polinómicas, exponenciales y logarítmicas hasta con un máximo de 6 incógnitas. En base a los datos obtenidos anteriormente, se ha realizado el ajuste por mínimos cuadrados de los coeficientes de las distintas funciones, empleando para ello la librería de cálculo numérico GNU GSL (Galassi et al., 2010), programada en C. Se ha utilizado un algoritmo multiparamétrico y no lineal tipo Levenberg-Marquardt, estableciéndose el criterio de convergencia en un error relativo de $10^{-9}$ entre dos iteraciones del resolvedor. Todo el proceso de tratamiento de datos y ajuste de expresiones se ha realizado implementando el citado algoritmo en un programa escrito en $\mathrm{C}++$.

La formulación completa del problema es extensa por la cantidad de casos existentes, pues se tienen 14 refrigerantes, 18 propiedades termodinámicas y 13 funciones, que hacen un total de 3,276 casos. Dada la imposibilidad de un reporte detallado para cada caso, se propone la siguiente metodología de cálculo:

1. Realizar el ajuste por mínimos cuadrados de todas las combinaciones posibles.

2. Para cada propiedad termodinámica de las listadas en la Tabla 3 se persigue obtener la función, de las mostradas en la Tabla 4, que minimice el error para todos los refrigerantes. El error está definido de acuerdo a la ecuación (2).

3. En base a las funciones elegidas para cada propiedad termodinámica según la Tabla 4 , se reportarán los respectivos coeficientes y se realizará un análisis del error de ajuste para cada propiedad y refrigerante considerados en el presente estudio.

Tabla 4: Funciones propuestas para las distintas propiedades termodinámicas en función de la temperatura de saturación.

\begin{tabular}{|c|c|c|}
\hline \multicolumn{3}{|c|}{ Función y número de parámetros } \\
\hline 1 & $f_{1}(T)=A+B T+C T^{2}$ & 3 \\
\hline 2 & $f_{2}(T)=A+B T+C T^{2}+D T^{3}$ & 4 \\
\hline 3 & $f_{3}(T)=A+B T+C T^{2}+D T^{3}+E T^{4}$ & 5 \\
\hline 4 & $\begin{aligned} f_{4}(T)=A+B T+ & C T^{2}+D T^{3}+E T^{4} \\
& +F T^{5}\end{aligned}$ & 6 \\
\hline 5 & $f_{5}(T)=A+B T+C T^{2}+D T^{3}+\frac{E}{T}+\frac{F}{T^{2}}$ & 6 \\
\hline 6 & $f_{5}(T)=A+B T+C T^{2}+\frac{D}{T}+\frac{E}{T^{2}}+\frac{F}{T^{3}}$ & 6 \\
\hline 7 & $f_{7}(T)=(A+B T) e^{(C+D T)}$ & 4 \\
\hline 8 & $f_{8}(T)=\left(A+B T+C T^{2}\right) e^{(D+E T)}$ & 5 \\
\hline 9 & $f_{9}(T)=(A+B T) e^{\left(C+D T+\frac{E}{T}\right)}$ & 5 \\
\hline 10 & $f_{10}(T)=(A+B T) e^{\left(C+D T+\frac{E}{T}+F \ln (T)\right)}$ & 6 \\
\hline 11 & $f_{11}(T)=e^{\left(A+\frac{B}{T}+C \ln (T)+D T+E T^{2}+\frac{F}{T^{2}}\right)}$ & 6 \\
\hline 12 & $\begin{array}{l}f_{12}(T)=A+B T^{(C+1)}+D T^{(C+2)} \\
\quad+E T^{(C+3)}+F T^{(C+4)}\end{array}$ & 6 \\
\hline 13 & $f_{13}(T)=(A+B T) e^{\left(C+D T+\frac{E}{T}+F T^{2}\right)}$ & 6 \\
\hline
\end{tabular}

\section{RESULTADOS}

Los resultados del proceso de ajuste para los distintos refrigerantes y propiedades se discuten en esta sección. Para dicho propósito se ha incluido la Tabla 5, en que se muestra el error relativo medio para las distintas funciones y propiedades. La primera observación es que el error relativo medio es bajo, del orden de $10^{-4}$. Teniendo en cuenta que el error relativo medio para la ecuación de 
estado de Peng-Robinson es del orden de $10^{-2}$, es un indicativo que es más interesante el empleo de correlaciones específicas para saturación que el de ecuaciones de estado generales.

Además, la función óptima para el mayor número de casos (12) es un polinomio de quinto orden. La función exponencial (11) también minimiza el error para cinco de propiedades termodinámicas, mientras que la función (13) únicamente lo hace para una. Es de resaltar que estas tres funciones son de seis parámetros. Como era de esperar, añadir más términos a una serie polinómica disminuye el error de ajuste. En lo relativo a las funciones exponenciales, no existe un patrón identificable entre las distintas expresiones propuestas, existiendo incluso comportamientos opuestos para distintas propiedades termodinámicas tal y como se observa para las funciones 7 a 10.

Tabla 5. Error de ajuste para cada propiedad termodinámica para todos los refrigerantes considerados para cada una de las funciones de ajuste consideradas. Se muestra resaltada la función que minimiza el error para cada propiedad termodinámica.

\begin{tabular}{cccccccccc}
\hline \multirow{2}{*}{ Función } & \multicolumn{8}{c}{ Propiedad termodinámica de la fracción líquida } \\
& pliquido & p papor & uliquido & hliquido & Sliquido & cvlíquido & cplíquido & SSliquido & $\mu J T$,liquido \\
\hline 1 & $2.8 \mathrm{E}-01$ & $2.9 \mathrm{E}-01$ & $3.1 \mathrm{E}-03$ & $3.7 \mathrm{E}-03$ & $2.8 \mathrm{E}-03$ & $4.0 \mathrm{E}-03$ & $5.3 \mathrm{E}-02$ & $5.3 \mathrm{E}-03$ & $1.2 \mathrm{E}+00$ \\
2 & $2.1 \mathrm{E}-02$ & $2.3 \mathrm{E}-02$ & $1.1 \mathrm{E}-03$ & $1.3 \mathrm{E}-03$ & $6.7 \mathrm{E}-04$ & $2.1 \mathrm{E}-03$ & $3.6 \mathrm{E}-02$ & $1.5 \mathrm{E}-03$ & $9.9 \mathrm{E}-01$ \\
3 & $1.6 \mathrm{E}-03$ & $2.2 \mathrm{E}-03$ & $5.1 \mathrm{E}-04$ & $5.7 \mathrm{E}-04$ & $3.3 \mathrm{E}-04$ & $1.2 \mathrm{E}-03$ & $2.4 \mathrm{E}-02$ & $8.0 \mathrm{E}-04$ & $3.5 \mathrm{E}-01$ \\
4 & $5.5 \mathrm{E}-04$ & $8.5 \mathrm{E}-04$ & $2.5 \mathrm{E}-04$ & $2.8 \mathrm{E}-04$ & $1.6 \mathrm{E}-04$ & $7.7 \mathrm{E}-04$ & $1.7 \mathrm{E}-02$ & $4.0 \mathrm{E}-04$ & $3.1 \mathrm{E}-01$ \\
5 & $8.8 \mathrm{E}-04$ & $1.3 \mathrm{E}-03$ & $3.4 \mathrm{E}-04$ & $3.8 \mathrm{E}-04$ & $2.1 \mathrm{E}-04$ & $9.4 \mathrm{E}-04$ & $1.9 \mathrm{E}-02$ & $5.3 \mathrm{E}-04$ & $4.1 \mathrm{E}-01$ \\
6 & $1.9 \mathrm{E}-03$ & $2.5 \mathrm{E}-03$ & $3.9 \mathrm{E}-04$ & $4.5 \mathrm{E}-04$ & $2.5 \mathrm{E}-04$ & $1.0 \mathrm{E}-03$ & $2.1 \mathrm{E}-02$ & $6.2 \mathrm{E}-04$ & $4.5 \mathrm{E}-01$ \\
7 & $1.6 \mathrm{E}-01$ & $1.6 \mathrm{E}-01$ & $1.5 \mathrm{E}-01$ & $3.6 \mathrm{E}-01$ & $7.4 \mathrm{E}-02$ & $6.4 \mathrm{E}-01$ & $7.8 \mathrm{E}-02$ & $5.0 \mathrm{E}-01$ & $8.3 \mathrm{E}-01$ \\
8 & $8.8 \mathrm{E}-01$ & $9.1 \mathrm{E}-01$ & $8.0 \mathrm{E}-01$ & $7.9 \mathrm{E}-01$ & $4.7 \mathrm{E}-01$ & $7.7 \mathrm{E}-01$ & $6.7 \mathrm{E}-01$ & $8.3 \mathrm{E}-01$ & $1.5 \mathrm{E}+00$ \\
9 & $3.1 \mathrm{E}-03$ & $3.8 \mathrm{E}-03$ & $2.3 \mathrm{E}-01$ & $7.8 \mathrm{E}-02$ & $1.7 \mathrm{E}-02$ & $7.1 \mathrm{E}-01$ & $7.2 \mathrm{E}-01$ & $5.0 \mathrm{E}-01$ & $3.0 \mathrm{E}-01$ \\
10 & $6.5 \mathrm{E}-02$ & $6.5 \mathrm{E}-02$ & $4.0 \mathrm{E}-01$ & $5.1 \mathrm{E}-01$ & $1.8 \mathrm{E}-02$ & $7.1 \mathrm{E}-01$ & $7.9 \mathrm{E}-01$ & $5.7 \mathrm{E}-01$ & $5.2 \mathrm{E}-01$ \\
11 & $1.3 \mathrm{E}-04$ & $2.2 \mathrm{E}-04$ & $2.4 \mathrm{E}-03$ & $2.5 \mathrm{E}-03$ & $2.3 \mathrm{E}-03$ & $9.0 \mathrm{E}-04$ & $1.3 \mathrm{E}-02$ & $2.2 \mathrm{E}-03$ & $9.6 \mathrm{E}-01$ \\
12 & $7.1 \mathrm{E}-02$ & $7.2 \mathrm{E}-02$ & $4.5 \mathrm{E}-04$ & $4.9 \mathrm{E}-04$ & $3.2 \mathrm{E}-04$ & $1.0 \mathrm{E}-03$ & $2.0 \mathrm{E}-02$ & $3.3 \mathrm{E}-02$ & $3.8 \mathrm{E}-01$ \\
13 & $3.6 \mathrm{E}-04$ & $6.0 \mathrm{E}-04$ & $7.2 \mathrm{E}-02$ & $8.3 \mathrm{E}-04$ & $4.8 \mathrm{E}-04$ & $6.4 \mathrm{E}-01$ & $7.2 \mathrm{E}-01$ & $9.3 \mathrm{E}-03$ & $1.9 \mathrm{E}-01$ \\
\hline Mínimo & $1.3 \mathrm{E}-04$ & $2.2 \mathrm{E}-04$ & $2.5 \mathrm{E}-04$ & $2.8 \mathrm{E}-04$ & $1.6 \mathrm{E}-04$ & $7.7 \mathrm{E}-04$ & $1.3 \mathrm{E}-02$ & $4.0 \mathrm{E}-04$ & $1.9 \mathrm{E}-01$ \\
\hline
\end{tabular}

\begin{tabular}{cccccccccc}
\hline \multirow{2}{*}{ Función } & \multicolumn{9}{c}{ Propiedad termodinámica de la fracción vapor } \\
& $\rho_{\text {liquido }}$ & $\rho_{\text {vapor }}$ & $\mathrm{u}_{\text {vapor }}$ & $\mathrm{h}_{\text {vapor }}$ & $\mathrm{S}_{\text {vapor }}$ & $\mathrm{cv}_{\text {vapor }}$ & $\mathrm{cp}_{\text {vapor }}$ & SS $_{\text {vapor }}$ & $\mu_{\text {JT,vapor }}$ \\
\hline 1 & $4.9 \mathrm{E}-03$ & $4.7 \mathrm{E}-01$ & $2.2 \mathrm{E}-03$ & $2.6 \mathrm{E}-03$ & $2.5 \mathrm{E}-03$ & $5.0 \mathrm{E}-03$ & $1.1 \mathrm{E}-01$ & $3.8 \mathrm{E}-03$ & $4.1 \mathrm{E}-02$ \\
2 & $1.9 \mathrm{E}-03$ & $1.4 \mathrm{E}-01$ & $8.1 \mathrm{E}-04$ & $9.1 \mathrm{E}-04$ & $5.2 \mathrm{E}-04$ & $2.4 \mathrm{E}-03$ & $7.6 \mathrm{E}-02$ & $8.0 \mathrm{E}-04$ & $9.1 \mathrm{E}-03$ \\
3 & $9.0 \mathrm{E}-04$ & $5.8 \mathrm{E}-02$ & $3.7 \mathrm{E}-04$ & $4.2 \mathrm{E}-04$ & $2.9 \mathrm{E}-04$ & $1.1 \mathrm{E}-03$ & $5.1 \mathrm{E}-02$ & $3.2 \mathrm{E}-04$ & $3.1 \mathrm{E}-03$ \\
4 & $4.6 \mathrm{E}-04$ & $2.7 \mathrm{E}-02$ & $1.8 \mathrm{E}-04$ & $2.0 \mathrm{E}-04$ & $1.3 \mathrm{E}-04$ & $5.9 \mathrm{E}-04$ & $3.5 \mathrm{E}-02$ & $1.4 \mathrm{E}-04$ & $6.9 \mathrm{E}-04$ \\
5 & $6.2 \mathrm{E}-04$ & $3.8 \mathrm{E}-02$ & $2.5 \mathrm{E}-04$ & $2.8 \mathrm{E}-04$ & $1.8 \mathrm{E}-04$ & $7.7 \mathrm{E}-04$ & $4.1 \mathrm{E}-02$ & $2.0 \mathrm{E}-04$ & $7.9 \mathrm{E}-04$ \\
6 & $7.2 \mathrm{E}-04$ & $4.6 \mathrm{E}-02$ & $2.9 \mathrm{E}-04$ & $3.3 \mathrm{E}-04$ & $2.1 \mathrm{E}-04$ & $8.9 \mathrm{E}-04$ & $4.4 \mathrm{E}-02$ & $2.5 \mathrm{E}-04$ & $8.7 \mathrm{E}-04$ \\
7 & $4.2 \mathrm{E}-03$ & $6.2 \mathrm{E}-02$ & $6.4 \mathrm{E}-01$ & $7.1 \mathrm{E}-01$ & $6.4 \mathrm{E}-01$ & $7.5 \mathrm{E}-03$ & $1.6 \mathrm{E}-01$ & $5.0 \mathrm{E}-01$ & $1.4 \mathrm{E}-01$ \\
8 & $5.7 \mathrm{E}-01$ & $2.3 \mathrm{E}+00$ & $9.3 \mathrm{E}-01$ & $9.3 \mathrm{E}-01$ & $9.0 \mathrm{E}-01$ & $6.4 \mathrm{E}-01$ & $6.5 \mathrm{E}-01$ & $6.9 \mathrm{E}-01$ & $3.0 \mathrm{E}-01$ \\
9 & $1.9 \mathrm{E}-03$ & $7.1 \mathrm{E}-02$ & $1.4 \mathrm{E}-01$ & $2.1 \mathrm{E}-01$ & $5.0 \mathrm{E}-01$ & $1.5 \mathrm{E}-01$ & $5.5 \mathrm{E}-01$ & $5.0 \mathrm{E}-01$ & $8.2 \mathrm{E}-02$ \\
10 & $8.4 \mathrm{E}-04$ & $1.3 \mathrm{E}-01$ & $6.4 \mathrm{E}-01$ & $5.7 \mathrm{E}-01$ & $5.7 \mathrm{E}-01$ & $2.4 \mathrm{E}-03$ & $6.0 \mathrm{E}-01$ & $6.4 \mathrm{E}-01$ & $4.7 \mathrm{E}-03$ \\
11 & $9.8 \mathrm{E}-04$ & $8.6 \mathrm{E}-03$ & $2.8 \mathrm{E}-04$ & $3.2 \mathrm{E}-04$ & $2.2 \mathrm{E}-04$ & $6.4 \mathrm{E}-04$ & $1.9 \mathrm{E}-02$ & $3.7 \mathrm{E}-04$ & $1.1 \mathrm{E}-03$ \\
12 & $7.1 \mathrm{E}-04$ & $3.6 \mathrm{E}-02$ & $3.0 \mathrm{E}-04$ & $3.4 \mathrm{E}-04$ & $4.6 \mathrm{E}-03$ & $9.9 \mathrm{E}-04$ & $3.8 \mathrm{E}-02$ & $2.6 \mathrm{E}-04$ & $3.5 \mathrm{E}-03$ \\
13 & $3.5 \mathrm{E}-03$ & $1.0 \mathrm{E}-01$ & $7.2 \mathrm{E}-02$ & $7.3 \mathrm{E}-02$ & $7.8 \mathrm{E}-04$ & $5.0 \mathrm{E}-01$ & $5.3 \mathrm{E}-01$ & $2.0 \mathrm{E}-03$ & $7.3 \mathrm{E}-02$ \\
\hline Mínimo & $4.6 \mathrm{E}-04$ & $8.6 \mathrm{E}-03$ & $1.8 \mathrm{E}-04$ & $2.0 \mathrm{E}-04$ & $1.3 \mathrm{E}-04$ & $5.9 \mathrm{E}-04$ & $1.9 \mathrm{E}-02$ & $1.4 \mathrm{E}-04$ & $6.9 \mathrm{E}-04$ \\
\hline
\end{tabular}

Una discusión en más profundidad puede realizarse si se considera el error relativo para cada temperatura y refrigerante. Este procedimiento da una estimación del error local, mientras que el obtenido de la Tabla 5 se puede considerar como un error medio en todo el intervalo. Dichas gráficas se han incluido en la Figura 2 (gráficas de la $2 \mathrm{a}$ a la $2 \mathrm{r}$ ) para las diferentes propiedades termodinámicas consideradas y la función 4 , que es la que minimiza el error para un mayor número de casos. Los siguientes comentarios se pueden realizar con relación a dicha Figura.

1. En el caso de la presión, como se comentó anteriormente, para los fluidos zeotrópicos existe diferencia entre la presión de líquido y vapor para una misma temperatura, de aquí el hecho de que únicamente se incluyan en la Figura $2 \mathrm{~b}$ la familia de los R4xx, pues para el resto de refrigerantes el gráfico sería idéntico al mostrado en la Figura 2a. 


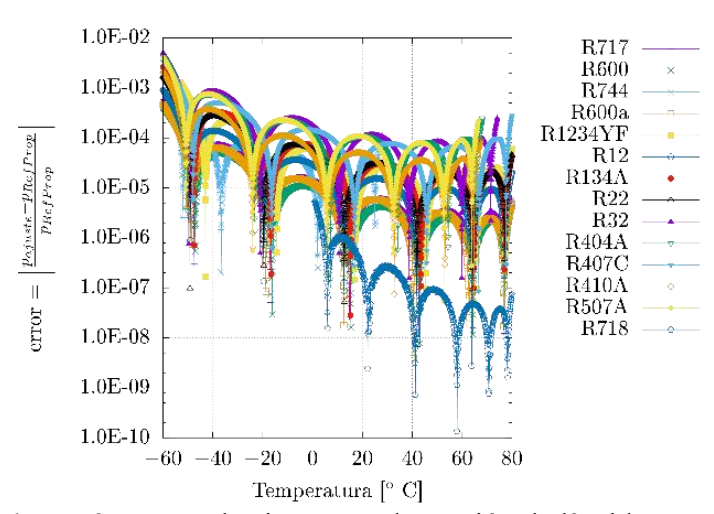

Figura 2a. Error de ajuste para la presión de líquido para la función 11. Todos los refrigerantes.

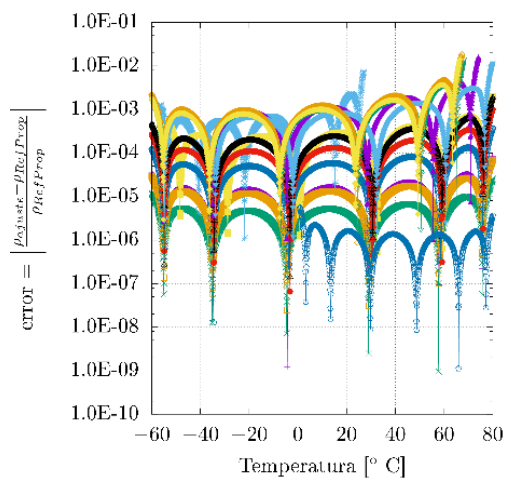

Figura 2c. Error de ajuste para la densidad de líquido para la función 4 . Todos los refrigerantes.

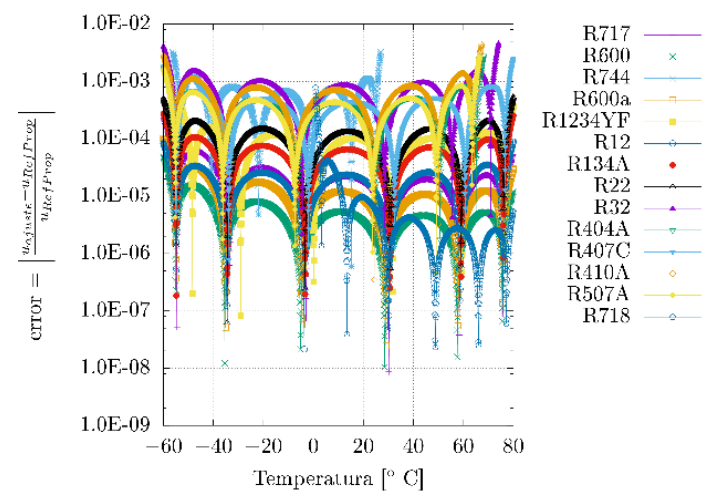

Figura 2e. Error de ajuste para la energía interna de líquido para la función 4 . Todos los refrigerantes.

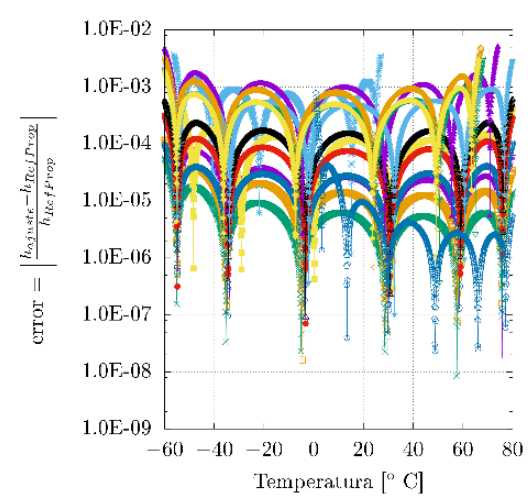

Figura 2g. Error de ajuste para la entalpía de líquido para la función 4 . Todos los refrigerantes.

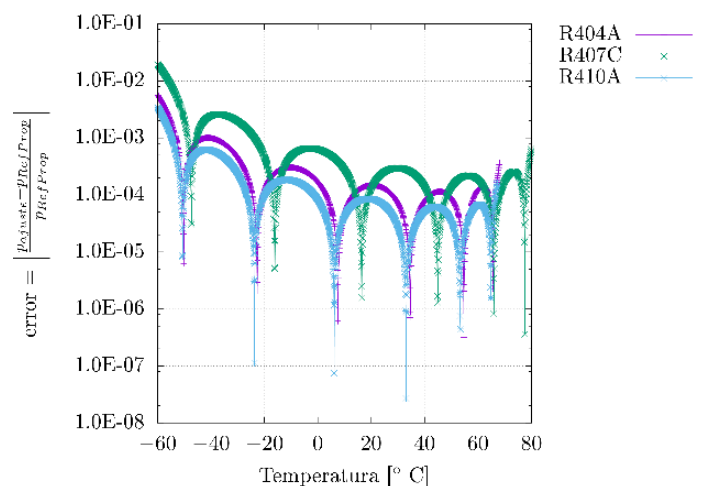

Figura 2b. Error de ajuste para la presión de vapor para la función 11. Refrigerantes zeotrópicos serie R4xx.

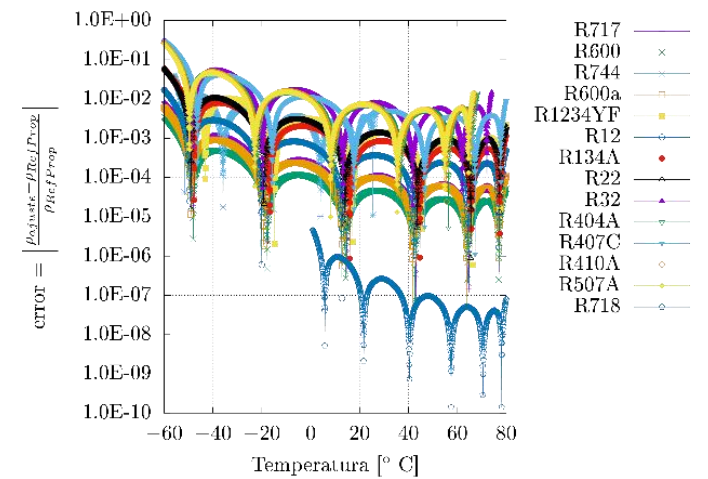

Figura 2d. Error de ajuste para la densidad de vapor para la función 11. Todos los refrigerantes.

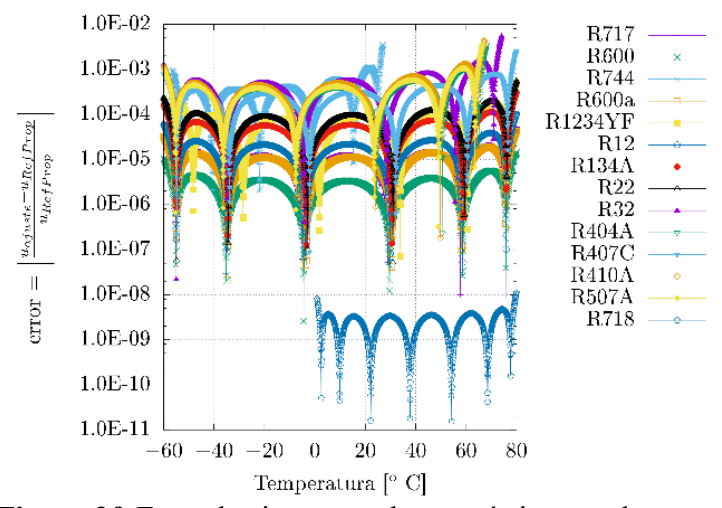

Figura 2f. Error de ajuste para la energía interna de vapor para la función 4 . Todos los refrigerantes.

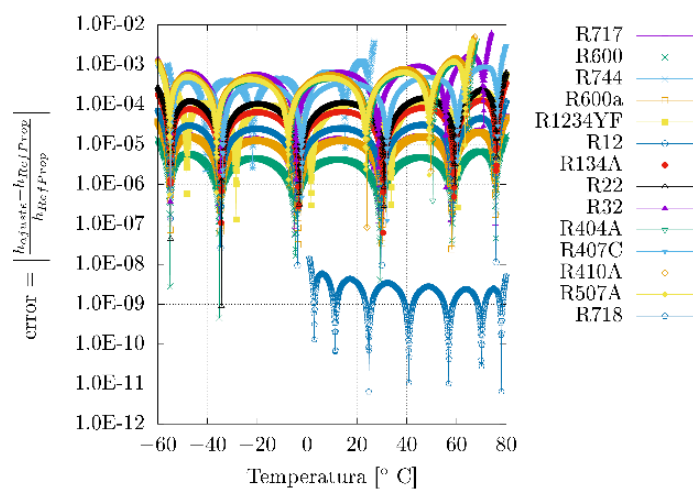

Figura 2 h. Error de ajuste para la entalpía de vapor para la función 4. Todos los refrigerantes. 


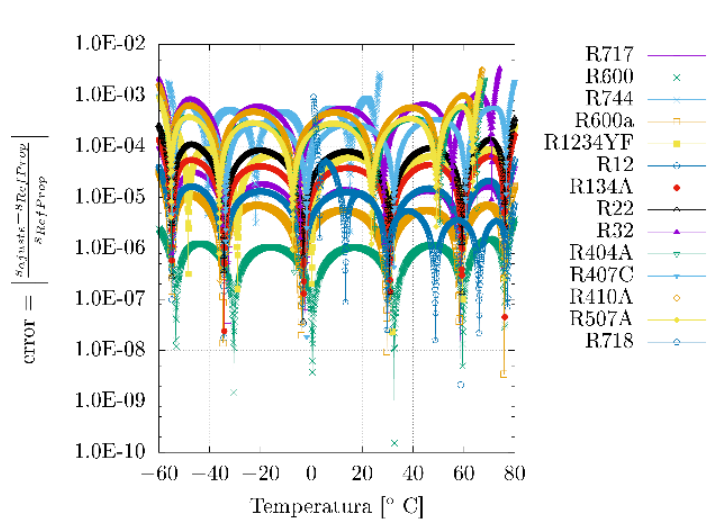

Figura 2i. Error de ajuste para la entropía de líquido para la función 4. Todos los refrigerantes.

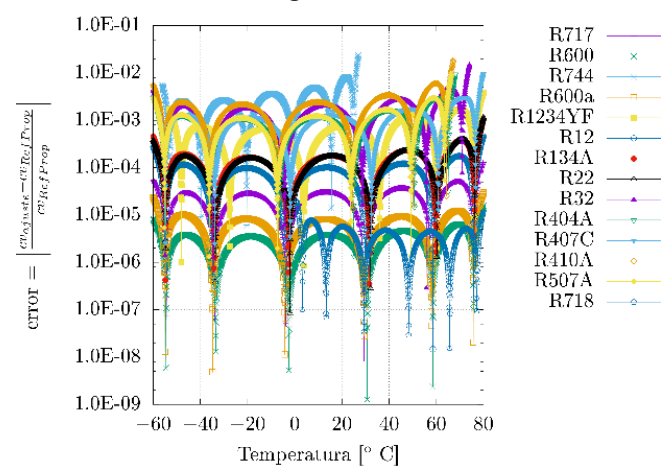

Figura 2k. Error de ajuste para el cv de líquido para la función 4. Todos los refrigerantes.
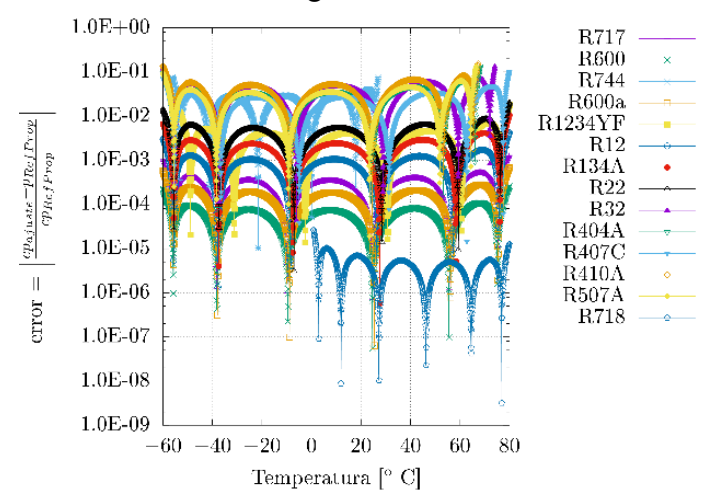

Figura $2 \mathbf{m}$. Error de ajuste para el cp de líquido para la función 11. Todos los refrigerantes.

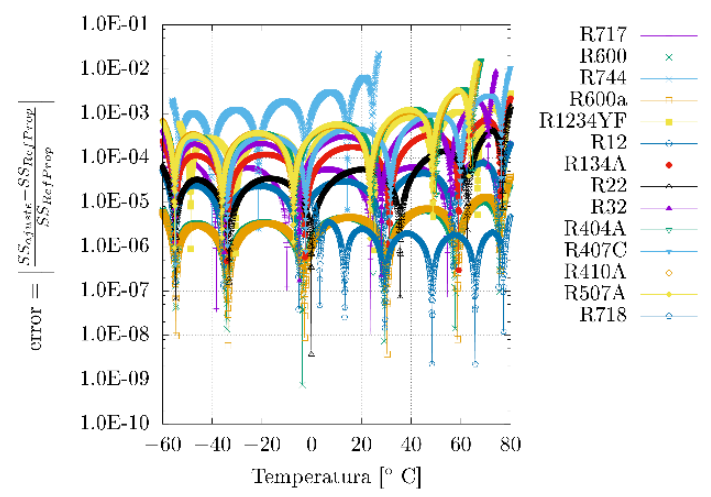

Figura 2o. Error de ajuste para la velocidad del sonido de líquido para la función 4 . Todos los refrigerantes.

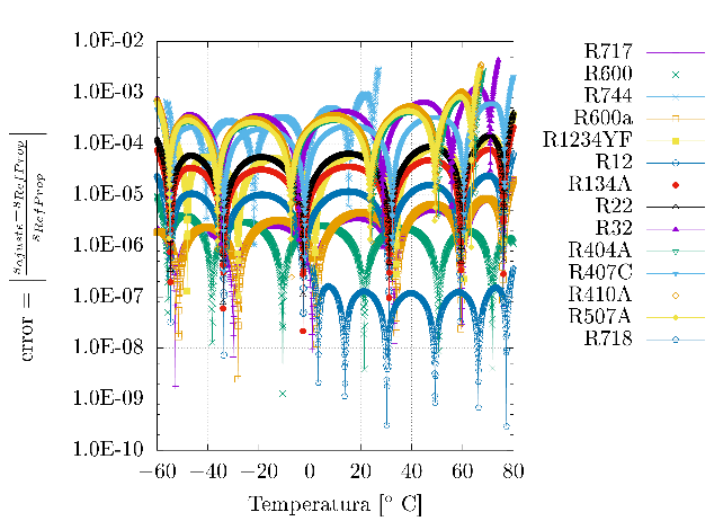

Figura 2j. Error de ajuste para la entropía de vapor para la función 4. Todos los refrigerantes.

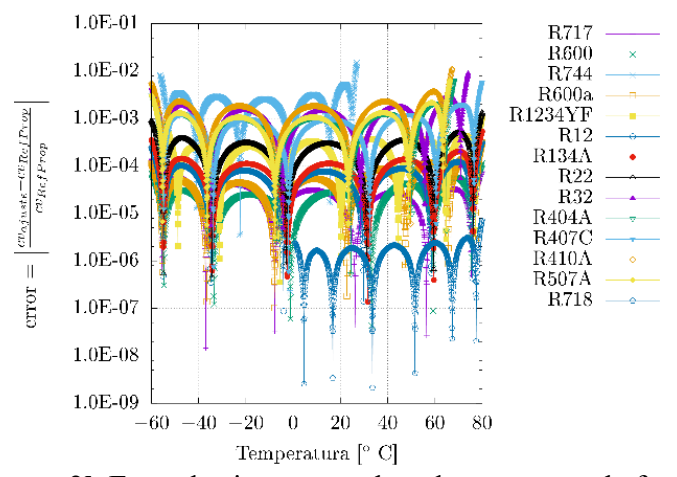

Figura 2l. Error de ajuste para el cv de vapor para la función 4. Todos los refrigerantes.

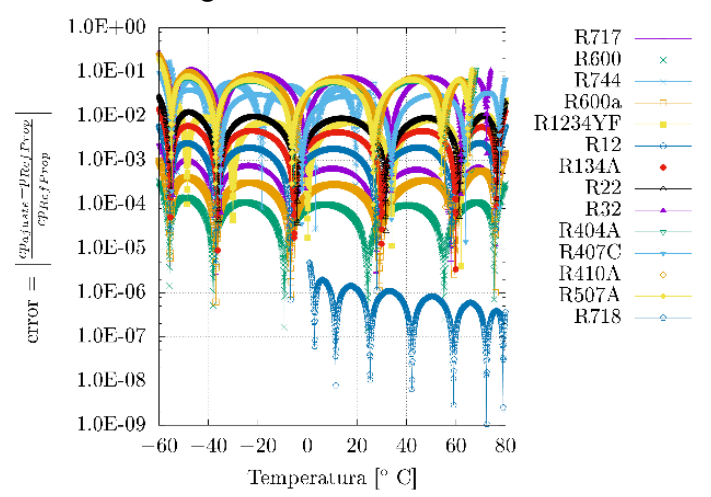

Figura 2n. Error de ajuste para el cp de vapor para la función 11. Todos los refrigerantes.

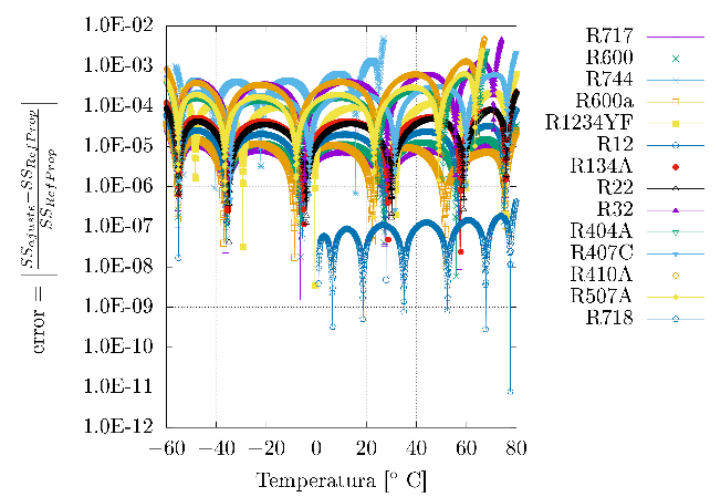

Figura 2p. Error de ajuste para la velocidad de sonido de vapor para la función 4 . Todos los refrigerantes. 


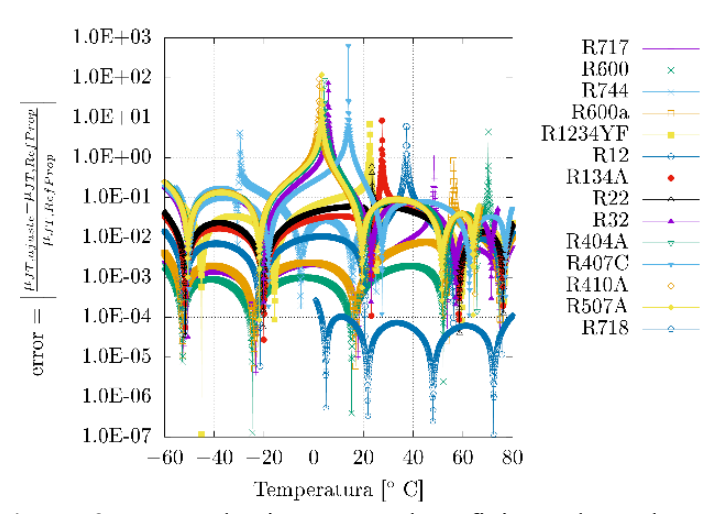

Figura 2q. Error de ajuste para el coeficiente de JouleThomson de líquido para la función 13. Todos los refrigerantes.

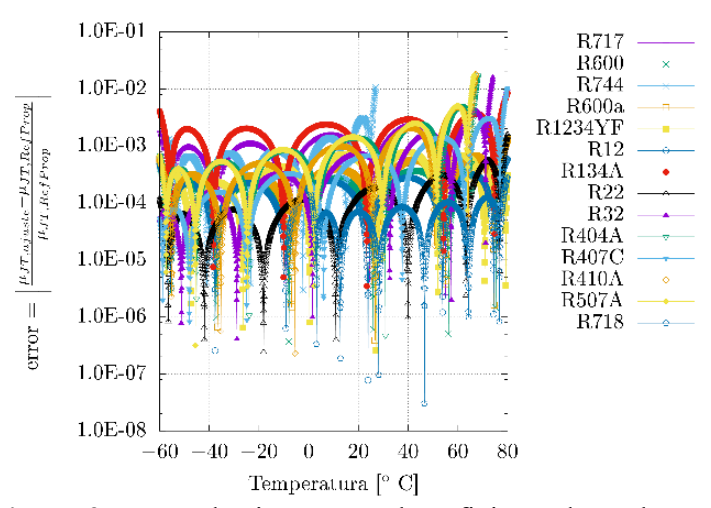

Figura 2r. Error de ajuste para el coeficiente de JouleThomson de vapor para la función 4 . Todos los refrigerantes.

Figura 2. Error de ajuste de para las distintas propiedades termodinámicas y refrigerantes.

2. La morfología de todas las curvas responde al hecho de que se ha tomado el valor absoluto en la ecuación de error, siendo cada mínimo de las curvas un paso por cero del error.

3. Globalmente, el comportamiento de cada función para cada uno de los refrigerantes es similar, encontrándose los pasos por cero aproximadamente en las mismas temperaturas. Se puede observar que el número de pasos por cero coincide con el número de parámetros de la función de ajuste, es decir, seis. Aquí hay que detallar dos peculiaridades. La primera es que, para el agua, dado que el intervalo de temperaturas considerado es inferior que para el resto de refrigerantes, el error cometido en el ajuste es por regla general menor. Relacionada con ésta, para los refrigerantes R744, R404a, R410a y R507a existe una ligera desviación hacia la temperatura de punto crítico (fin de las curvas). El tratamiento del punto crítico es un fenómeno complejo, pues tanto la medición de propiedades, así como el ajuste de ecuaciones se convierten en problemas no triviales (Span, 2000).

4. No se pueden observar tendencias que relacionen el error con la temperatura, de lo que se deduce que la predicción de las propiedades oscila alrededor del valor obtenido de RefProp, siendo la amplitud de dichas oscilaciones simétrica e independiente de la temperatura.

5. También se puede observar que, para las distintas funciones termodinámicas, el error cometido para los distintos refrigerantes lleva casi siempre el mismo orden. Así, por ejemplo, se puede observar que para el R600 y el R600a se encuentra, para la mayor parte de las gráficas, errores bajos, mientras que el R32 y el R407c siempre tienen errores altos. Este fenómeno brinda una idea novedosa en el sentido de que la dificultad de ajuste de una propiedad no es aislada, sino que es traducible a todas las propiedades termodinámicas del fluido considerado.

6. El comportamiento de la energía interna y entalpía de líquido es muy similar debido a que dichas propiedades toman valores similares a lo largo de todo el rango de temperaturas.
7. El comportamiento de la curva de error para el coeficiente de Joule-Thomson para líquido es peculiar, existiendo errores relativos anormalmente altos. El motivo es que dicho coeficiente pasa de valores negativos $-\mathrm{a}$ bajas temperaturas- a valores positivos $-\mathrm{a}$ altas temperaturas. La transición por cero crea una singularidad en la ecuación (1), motivo por el cual aparecen los altos valores de error relativo en la Figura 2q.

Dado que la función 4 es la que minimiza el error para el mayor número de propiedades, se ha elegido ésta para mostrar los seis coeficientes de ajuste para las distintas propiedades de cada fluido en el Anexo A, con un valor de 5 cifras significativas. El Anexo A es una herramienta de utilidad, ya que condensa los productos de la presente investigación en unos coeficientes que, aplicados a la morfología de la función 4, permiten obtener datos termodinámicos para 14 refrigerantes y 18 propiedades termodinámicas. La incertidumbre media para cada una de estas ecuaciones se muestra en la Tabla 5, mientras que la estimación local se puede obtener a partir de las Figuras 2a a 2 r.

\section{CONCLUSIONES}

En la presente investigación se ha escogido la expresión óptima entre una base de funciones propuestas para el ajuste de diferentes propiedades termodinámicas en saturación de refrigerantes comunes. Se ha comprobado que el uso de funciones específicas para saturación da mejores resultados que ecuaciones de estado cúbicas tipo Peng-Robinson y derivados que modelan todo el diagrama p-v-T. Globalmente se ha encontrado que las mejores funciones son polinomios puros o expresiones tipo exponenciales. La incertidumbre alcanzada en el proceso de ajuste es satisfactoria, encontrándose errores relativos promedios del orden de $10^{-4}$. El análisis de la incertidumbre local ha demostrado que ésta es oscilatoria a lo largo del rango de temperatura considerado, siendo su valor función del refrigerante considerado. Además, se ha encontrado que la dificultad para ajustar una propiedad termodinámica de un refrigerante no es aislada, sino que, por regla general, está asociada a ajustes menos 
significativos para el resto de propiedades termodinámicas.

\section{AGRADECIMIENTOS}

Se agradece a la "Dirección de Investigación y DesarrolloUTA" por proporcionar financiamiento para el desarrollo de la presente investigación a través del proyecto 2441 CU-P-2015.

\section{POLÍTICA DE REPRODUCIBILIDAD}

El autor de este trabajo tiene la política de hacer los códigos empleados abiertos. Es por ello que las rutinas en $\mathrm{C}++$ empleadas para resolver las ecuaciones de PengRobinson están disponibles en línea a través de Mendeley bajo licencia CC-BY-4.0 (García del Valle, 2018).

\section{NOMENCLATURA}

$\mathrm{Cp}$ calor específico a presión constante $\left[\mathrm{kJ} \mathrm{kg}^{-1} \mathrm{~K}^{-1}\right]$

$\mathrm{Cv}$ calor específico a volumen constante $\left[\mathrm{kJ} \mathrm{kg}^{-1} \mathrm{~K}^{-1}\right]$

$\mathrm{H}$ entalpía $\left[\mathrm{kJ} \mathrm{kg}^{-1}\right]$

$\mathrm{P}$ presión $[\mathrm{kPa}]$

$\mathrm{U} \quad$ energía interna $\left[\mathrm{kJ} \mathrm{kg}^{-1}\right]$

$\mathrm{S}$ entropía $\left[\mathrm{kJ} \mathrm{kg}^{-1} \mathrm{~K}^{-1}\right]$

$\mathrm{T}$ temperatura $[\mathrm{K}]$

$\mathrm{V} \quad$ volumen específico $\left[\mathrm{m}^{3} \mathrm{~kg}^{-1}\right]$

SS velocidad del sonido $\left[\mathrm{m} \mathrm{s}^{-1}\right]$

$\mu_{\mathrm{JT}}$ coeficiente de Joule-Thomson $\left[\mathrm{K} \mathrm{Pa}^{-1}\right]$

$\rho \quad$ densidad $\left[\mathrm{kg} \mathrm{m}^{3}\right]$

\section{BIBLIOGRAFÍA}

Affandia, M., Mamat, N., Kanafiah, S. N. \& Khalid, N. S. (2013). Simplified equations for saturated steam properties for simulation purposes. Procedia Engineering, 53, 722-726.

Atalay, H. \& Coban, M. T. (2015). Modeling of thermodynamic properties for pure refrigerants and refrigerant mixtures by using the Helmholtz equation of state and cubic spline curve fitting method. Universal Journal of Mechanical Engineering, 3(6), 229-251.

Bell, I. H., Wronski, J., Quoilin, S. \& Lemort, V. (2014). Pure and pseudo-pure fluid thermophysical property evaluation and the open-source thermophysical property library CoolProp. Industrial \& Engineering Chemistry Research, 53(6), 2498-2508.

Brown, J. S. (2007). Predicting performance of refrigerants using the Peng-Robinson Equation of State. International Journal of Refrigeration, 30(8), 13191328.

CAREL Industries S.p.A. (2017). Sistemas de válvulas de expansión electrónicas a pasos y controlador de recalentamiento de gas. Descargado de www.carel.es/electronic-expansion-valve
Cleland, A. C. (1994). Polynomial curve-fits for refrigerant thermodynamic properties: extension to include R134a. International Journal of Refrigeration, 17(4), 245-249.

DuPont TM Suva ${ }^{\circledR}$ (2017). Thermodynamic properties of HFC-134a (1,1,1,2-tetrafluoroethane). Technical Bulletin. Descargado de https:/www.chemours.com/Refrigerants/en_US/ products/Freon/Freon-134a.html

Figueira, F. L., Lugo, L. \& Olivera-Fuentes, C. (2007). Generalized parameters of the Stryjek-Vera and Gibbons-Laughton cohesion functions for use with cubic EOS of the van der Waals type. Fluid Phase Equilibria, 259, 105-115.

Galassi, M., Davies, J., Theiler, J., Gough, B., Jungman, G., Alken, P., Booth, M. \& Rossi, F. (2010). GNU Scientific Library. Descargado de http://gnu.org/gsl

García del Valle, J. (2018). C++ program for computing the liquid-vapor equilibrium using cubic Equations of State, Mendeley Data, v1.

http://dx.doi.org/10.17632/st4dss5f22.1

Hewitt, N. J., McMullan, J. T., Evans, R. H. \& Mongoney, B. (1996). Martin-Hou, Peng-Robingson and Redlich-Kwong-Soave equations of state for HFC 125. International Journal of Energy Research, 20, $33-$ 39.

Iglesias-Silva, G. A., Miller, R. C., Ceballos, A. D., Hall, K. R. \& Holste, J. C. (1995). Accurate vapor pressure equation for refrigerants. Fluid Phase Equilibria, 111, 203-212.

Lopez-Echeverry, J. S., Reif-Acerman, S. \& AraujoLopez, E. (2017). Peng-Robinson equation of state: 40 years through cubics. Fluid Phase Equilibria, 447, 39 71.

NIST. (2017). Librería REFPROP “Refrigerants Properties" del National Institute of Standards (USA). Descargado de https://www.nist.gov/srd/refprop

Peng, D. Y. \& Robinson, D. B. (1976). A new two constant equation of state. Industrial \& Engineering Chemistry Fundamentals, 15(1), 59-64.

Peng, D. Y. \& Robinson, D. B. (1980). Two- and threephase equilibrium calculations for coal gasification and related processes. In: Newman, S. A., Barner, H. E., Klein, M. \& Sandler, S. I. (Eds.). Thermodynamics of aqueous systems with industrial applications. Washington, D.C., US: American Chemical Society, 393-414

Pérez Casas, J. M. (1987). La ecuación de estado de Peng-Robinson en la industria petroquímica. Ingeniería Química, 223, 461-465.

Poling, B. E., Prausnitz, J. M. \& O'Connell, J. P. (2001). The properties of gases and liquids, $5^{\text {th }} \mathrm{Ed}$. Nueva York, EE.UU.: McGrawHill, 803 pp.

Span, R. (2000). Multiparameter equations of state, an accurate source of thermodynamic property data. Bochum, Alemania: Springer, 367 pp.

Vikrant, A. \& Radermacher, R. (2014). Standardized Polynomials for Fast Evaluation of Refrigerant Thermophysical Properties. International Refrigeration and Air Conditioning Conference, Purdue University, Illinois, USA.

Wolfgang, W. \& Kretzschmar, H. J. (2008). International steam tables properties of water and steam based on 
the industrial formulation IAPWS-IF97IAPWS steam properties. Berlin, Alemania: Springer, 392 pp.

YellowJacket. (2017). Analizador/recuperador de gas para sistemas frigorificos. Descargado de:

http://yellowjacket.com/product/refrigeration-systemanalyzer
Zhao, D., Ding, G. \& Wu, Z. (2009). Extension of the implicit curve-fitting method for fast calculation of thermodynamic properties of refrigerants in supercritical region. International Journal of Refrigeration, 32(7), 1615-1625.

\section{ANEXO A}

Coeficientes de ajuste para la función 4 para las diferentes propiedades termodinámicas de los distintos refrigerantes. La forma de la ecuación 4 es: $f_{4}(T)=A+B T+C T^{2}+D T^{3}+E T^{4}+F T^{5}$. Las unidades de entrada son grados Kelvin y las de salida son las consideradas en la Tabla 3 para cada propiedad termodinámica.

\begin{tabular}{|c|c|c|c|c|c|c|c|}
\hline Refri & Propiedad & $\mathrm{A}$ & B & $\mathrm{C}$ & $\mathrm{D}$ & E & $\mathrm{F}$ \\
\hline \multirow{18}{*}{ R717 } & plíquido & $6,85929 \mathrm{E}+03$ & $-1,51099 \mathrm{E}+02$ & $1,27688 \mathrm{E}+00$ & $-5,03074 \mathrm{E}-03$ & $8,58622 \mathrm{E}-06$ & $-3,74710$ E-09 \\
\hline & $\mathrm{p}_{\text {vapor }}$ & $6,85929 \mathrm{E}+03$ & $-1,51099 \mathrm{E}+02$ & $1,27688 \mathrm{E}+00$ & $-5,03074 \mathrm{E}-03$ & 8,58622E-06 & $-3,74710$ E-09 \\
\hline & Plíquido & $1,28990 \mathrm{E}+03$ & $-9,00308 \mathrm{E}+00$ & 6,99612E-02 & $-2,98586 \mathrm{E}-04$ & 6,22496E-07 & $-5,20951 \mathrm{E}-10$ \\
\hline & $\rho_{\text {vapor }}$ & $-5,94073 \mathrm{E}+02$ & $1,13842 \mathrm{E}+01$ & -8,71797E-02 & $3,34827 \mathrm{E}-04$ & $-6,49990$ E-07 & $5,17616 \mathrm{E}-10$ \\
\hline & ulíquido & $-1,08597 \mathrm{E}+06$ & $1,12225 \mathrm{E}+04$ & $-6,80951 \mathrm{E}+01$ & $3,08557 \mathrm{E}-01$ & $-6,66636 \mathrm{E}-04$ & 5,69730E-07 \\
\hline & $\mathrm{h}_{\text {líquido }}$ & $-1,18030 \mathrm{E}+06$ & $1,30453 \mathrm{E}+04$ & $-8,22651 \mathrm{E}+01$ & $3,64336 \mathrm{E}-01$ & $-7,79043 \mathrm{E}-04$ & 6,63974E-07 \\
\hline & Slíquido & $-7,30836 \mathrm{E}+03$ & $6,83486 \mathrm{E}+01$ & $-2,80087 \mathrm{E}-01$ & $9,04809 \mathrm{E}-04$ & $-1,71870$ E-06 & 1,40454E-09 \\
\hline & cVlíquido & $2,06822 \mathrm{E}+03$ & $2,68777 \mathrm{E}+01$ & $-2,62531 \mathrm{E}-01$ & 1,17711E-03 & $-2,60454 \mathrm{E}-06$ & 2,29362E-09 \\
\hline & cplíquido & $-5,91495 \mathrm{E}+04$ & $1,17928 \mathrm{E}+03$ & $-8,87389 \mathrm{E}+00$ & $3,37186 \mathrm{E}-02$ & $-6,45426 \mathrm{E}-05$ & 4,98803E-08 \\
\hline & $\mathrm{SS}_{\text {líquido }}$ & $1,18401 \mathrm{E}+04$ & $-1,47300 \mathrm{E}+02$ & $9,37360 \mathrm{E}-01$ & $-3,13941 \mathrm{E}-03$ & 5,30046E-06 & $-3,64157 \mathrm{E}-09$ \\
\hline & $\mu_{\mathrm{JT}, \text { liquido }}$ & $-1,69927 \mathrm{E}-05$ & $3,18243 \mathrm{E}-07$ & $-2,42844 \mathrm{E}-09$ & $9,30350 \mathrm{E}-12$ & $-1,78864 \mathrm{E}-14$ & 1,38629E-17 \\
\hline & $\mathrm{u}_{\text {vapor }}$ & $2,53104 \mathrm{E}+06$ & $-2,61492 \mathrm{E}+04$ & $2,11146 \mathrm{E}+02$ & $-7,97306 \mathrm{E}-01$ & $1,49642 \mathrm{E}-03$ & $-1,14575 \mathrm{E}-06$ \\
\hline & $h_{\text {vapor }}$ & $2,86671 \mathrm{E}+06$ & $-3,19781 \mathrm{E}+04$ & $2,57913 \mathrm{E}+02$ & $-9,67571 \mathrm{E}-01$ & 1,80512E-03 & $-1,38060$ E-06 \\
\hline & Svapor & $3,57966 \mathrm{E}+04$ & $-3,86441 \mathrm{E}+02$ & $2,19045 \mathrm{E}+00$ & $-6,68304 \mathrm{E}-03$ & 1,06676E-05 & -7,07854E-09 \\
\hline & $\mathrm{cV}_{\text {vapor }}$ & $-7,31339 \mathrm{E}+03$ & $1,77515 \mathrm{E}+02$ & $-1,40076 \mathrm{E}+00$ & 5,35907E-03 & $-9,84284 \mathrm{E}-06$ & 7,12248E-09 \\
\hline & $\mathrm{cp}_{\text {vapor }}$ & $-1,35992 \mathrm{E}+05$ & $2,65589 \mathrm{E}+03$ & $-2,03628 \mathrm{E}+01$ & 7,77123E-02 & $-1,47711 \mathrm{E}-04$ & $1,12462 \mathrm{E}-07$ \\
\hline & $\mathrm{SS}_{\text {vapor }}$ & $4,50021 \mathrm{E}+02$ & $-4,81673 \mathrm{E}+00$ & 4,60222E-02 & $-1,75340 \mathrm{E}-04$ & 3,20307E-07 & $-2,47882 \mathrm{E}-10$ \\
\hline & $\mu_{\mathrm{JT}, \mathrm{vapor}}$ & $9,05729 \mathrm{E}-03$ & -1,39844E-04 & $8,82111 \mathrm{E}-07$ & $-2,81762 \mathrm{E}-09$ & $4,53555 \mathrm{E}-12$ & $-2,93450 \mathrm{E}-15$ \\
\hline \multirow{18}{*}{ R600 } & plíquido & $2,41124 \mathrm{E}+03$ & $-5,13092 \mathrm{E}+01$ & $4,23808 \mathrm{E}-01$ & $-1,65934 \mathrm{E}-03$ & 2,91469E-06 & $-1,52695 \mathrm{E}-09$ \\
\hline & $\mathrm{p}_{\text {vapor }}$ & $2,41124 \mathrm{E}+03$ & $-5,13092 \mathrm{E}+01$ & $4,23808 \mathrm{E}-01$ & $-1,65934 \mathrm{E}-03$ & 2,91469E-06 & $-1,52695 \mathrm{E}-09$ \\
\hline & Plíquido & $1,08361 \mathrm{E}+03$ & $-5,29683 \mathrm{E}+00$ & 3,40331E-02 & $-1,32253 \mathrm{E}-04$ & 2,58622E-07 & $-2,10237 \mathrm{E}-10$ \\
\hline & $\rho_{\text {vapor }}$ & $-2,15817 \mathrm{E}+02$ & 4,08809E+00 & $-3,09600 \mathrm{E}-02$ & 1,18161E-04 & $-2,31162 \mathrm{E}-07$ & 1,91102E-10 \\
\hline & ulíquido & $-4,65160 \mathrm{E}+05$ & 4,09703E+03 & $-1,62053 \mathrm{E}+01$ & 5,93239E-02 & $-1,02893 \mathrm{E}-04$ & 7,80925E-08 \\
\hline & hlíquido & $-4,75107 \mathrm{E}+05$ & $4,28948 \mathrm{E}+03$ & $-1,77206 \mathrm{E}+01$ & 6,54857E-02 & $-1,16101 \mathrm{E}-04$ & $9,02950 \mathrm{E}-08$ \\
\hline & Slíquido & $-3,86241 \mathrm{E}+03$ & 4,19162E+01 & $-1,79372 \mathrm{E}-01$ & 5,05799E-04 & $-7,65460 \mathrm{E}-07$ & 4,92670E-10 \\
\hline & cVlíquido & $1,06594 \mathrm{E}+03$ & $1,14741 \mathrm{E}+01$ & $-1,23388 \mathrm{E}-01$ & 5,80464E-04 & $-1,17816 \mathrm{E}-06$ & $9,04000 \mathrm{E}-10$ \\
\hline & cplíquido & $-4,22034 \mathrm{E}+03$ & $1,22056 \mathrm{E}+02$ & $-9,65891 \mathrm{E}-01$ & 3,81400E-03 & $-7,40722 \mathrm{E}-06$ & 5,76354E-09 \\
\hline & SS líquido & $3,06472 \mathrm{E}+03$ & $-1,48271 \mathrm{E}+01$ & 6,91872E-02 & $-2,69670 \mathrm{E}-04$ & $5,44756 \mathrm{E}-07$ & $-4,46086 \mathrm{E}-10$ \\
\hline & $\mu_{\mathrm{JT}, \text { liquido }}$ & $-1,24668 \mathrm{E}-05$ & $2,30899 \mathrm{E}-07$ & $-1,79529 \mathrm{E}-09$ & $6,98851 \mathrm{E}-12$ & $-1,35821 \mathrm{E}-14$ & $1,06289 \mathrm{E}-17$ \\
\hline & $\mathrm{u}_{\text {vapor }}$ & $4,20401 \mathrm{E}+05$ & $-2,09594 \mathrm{E}+03$ & $2,20800 \mathrm{E}+01$ & $-7,95765 \mathrm{E}-02$ & $1,55465 \mathrm{E}-04$ & $-1,25707 \mathrm{E}-07$ \\
\hline & $\mathrm{h}_{\text {vapor }}$ & $4,69726 \mathrm{E}+05$ & $-2,84883 \mathrm{E}+03$ & $2,84283 \mathrm{E}+01$ & $-1,01567 \mathrm{E}-01$ & 1,93726E-04 & $-1,55110 \mathrm{E}-07$ \\
\hline & Svapor & $9,61289 \mathrm{E}+03$ & $-9,82335 \mathrm{E}+01$ & $5,46356 \mathrm{E}-01$ & $-1,57719 \mathrm{E}-03$ & 2,39015E-06 & $-1,51030$ E-09 \\
\hline & CVvapor & $7,74265 \mathrm{E}+02$ & $2,52828 \mathrm{E}+00$ & $-1,72890 \mathrm{E}-02$ & 1,06197E-04 & $-1,87034 \mathrm{E}-07$ & 1,12202E-10 \\
\hline & $c p_{\text {vapor }}$ & $-7,80016 \mathrm{E}+03$ & 1,70927E+02 & $-1,31484 \mathrm{E}+00$ & $5,09606 \mathrm{E}-03$ & $-9,78895 \mathrm{E}-06$ & 7,55808E-09 \\
\hline & $\mathrm{SS}_{\text {vapor }}$ & $2,72812 \mathrm{E}+02$ & $-3,11456 \mathrm{E}+00$ & 2,71312E-02 & $-1,00128 \mathrm{E}-04$ & 1,81159E-07 & $-1,40758 \mathrm{E}-10$ \\
\hline & $\mu \mathrm{JT}$, vapor & $2,50846 \mathrm{E}-03$ & $-3,51675 \mathrm{E}-05$ & $2,06161 \mathrm{E}-07$ & $-6,20101 \mathrm{E}-10$ & $9,48192 \mathrm{E}-13$ & $-5,85763 \mathrm{E}-16$ \\
\hline
\end{tabular}




\begin{tabular}{|c|c|c|c|c|c|c|c|}
\hline \multirow{18}{*}{ R744 } & plíquido & $-1,61910 \mathrm{E}+05$ & $3,25819 \mathrm{E}+03$ & $-2,60720 \mathrm{E}+01$ & $1,03847 \mathrm{E}-01$ & $-2,07503 \mathrm{E}-04$ & 1,70599E-07 \\
\hline & $\mathrm{p}_{\text {vapor }}$ & $-1,61910 \mathrm{E}+05$ & $3,25819 \mathrm{E}+03$ & $-2,60720 \mathrm{E}+01$ & 1,03847E-01 & $-2,07503 \mathrm{E}-04$ & 1,70599E-07 \\
\hline & ९líquido & $2,66629 \mathrm{E}+05$ & $-5,33560 \mathrm{E}+03$ & 4,28915E+01 & $-1,72251 \mathrm{E}-01$ & 3,45474E-04 & $-2,76982 \mathrm{E}-07$ \\
\hline & $\rho_{\text {vapor }}$ & $-2,62571 \mathrm{E}+05$ & $5,28851 \mathrm{E}+03$ & $-4,25391 \mathrm{E}+01$ & $1,70838 \mathrm{E}-01$ & $-3,42639 \mathrm{E}-04$ & 2,74711E-07 \\
\hline & ulíquido & $-4,65635 \mathrm{E}+07$ & $9,32418 \mathrm{E}+05$ & $-7,48006 \mathrm{E}+03$ & $3,00240 \mathrm{E}+01$ & $-6,01932 \mathrm{E}-02$ & 4,82440E-05 \\
\hline & $\mathrm{h}_{\text {líquido }}$ & $-5,33913 \mathrm{E}+07$ & $1,06998 \mathrm{E}+06$ & $-8,58706 \mathrm{E}+03$ & $3,44723 \mathrm{E}+01$ & $-6,91229 \mathrm{E}-02$ & $5,54143 \mathrm{E}-05$ \\
\hline & Slíquido & $-1,73392 \mathrm{E}+05$ & $3,45557 \mathrm{E}+03$ & $-2,76148 \mathrm{E}+01$ & 1,10590E-01 & $-2,21395 \mathrm{E}-04$ & 1,77242E-07 \\
\hline & cVlíquido & $-6,38627 \mathrm{E}+05$ & $1,27118 \mathrm{E}+04$ & $-1,00829 \mathrm{E}+02$ & 3,99114E-01 & $-7,88690 \mathrm{E}-04$ & $6,22669 \mathrm{E}-07$ \\
\hline & cplíquido & $-3,40015 \mathrm{E}+07$ & $6,79976 \mathrm{E}+05$ & $-5,42711 \mathrm{E}+03$ & $2,16106 \mathrm{E}+01$ & $-4,29348 \mathrm{E}-02$ & $3,40496 \mathrm{E}-05$ \\
\hline & $\mathrm{SS}_{\text {líquido }}$ & $2,02634 \mathrm{E}+05$ & $-3,92295 \mathrm{E}+03$ & $3,05776 \mathrm{E}+01$ & $-1,19158 \mathrm{E}-01$ & 2,31880E-04 & $-1,80428 \mathrm{E}-07$ \\
\hline & $\mu_{\mathrm{JT}, \text { liquido }}$ & $-6,61280 \mathrm{E}-03$ & $1,32968 \mathrm{E}-04$ & $-1,06763 \mathrm{E}-06$ & 4,27906E-09 & $-8,56204 \mathrm{E}-12$ & $6,84370 \mathrm{E}-15$ \\
\hline & $\mathrm{u}_{\mathrm{vapor}}$ & $6,62600 \mathrm{E}+07$ & $-1,32857 \mathrm{E}+06$ & $1,06936 \mathrm{E}+04$ & $-4,29579 \mathrm{E}+01$ & $8,61803 \mathrm{E}-02$ & $-6,91149 \mathrm{E}-05$ \\
\hline & $\mathrm{h}_{\text {vapor }}$ & 7,93067E+07 & $-1,59147 \mathrm{E}+06$ & $1,28122 \mathrm{E}+04$ & $-5,14743 \mathrm{E}+01$ & 1,03275E-01 & $-8,28366 \mathrm{E}-05$ \\
\hline & Svapor & $2,61836 \mathrm{E}+05$ & $-5,18184 \mathrm{E}+03$ & 4,14444E+01 & $-1,65879 \mathrm{E}-01$ & 3,31938E-04 & $-2,65686 \mathrm{E}-07$ \\
\hline & $\mathrm{cV}_{\text {vapor }}$ & $-8,62491 \mathrm{E}+05$ & $1,73123 \mathrm{E}+04$ & $-1,38660 \mathrm{E}+02$ & 5,54277E-01 & $-1,10584 \mathrm{E}-03$ & $8,81180 \mathrm{E}-07$ \\
\hline & $\mathrm{cp}_{\text {vapor }}$ & $-5,76756 \mathrm{E}+07$ & $1,15315 \mathrm{E}+06$ & $-9,20174 \mathrm{E}+03$ & $3,66332 \mathrm{E}+01$ & $-7,27635 \mathrm{E}-02$ & $5,76900 \mathrm{E}-05$ \\
\hline & $\mathrm{SS}_{\text {vapor }}$ & $3,48028 \mathrm{E}+04$ & $-6,94560 \mathrm{E}+02$ & $5,55965 \mathrm{E}+00$ & $-2,21807 \mathrm{E}-02$ & 4,41414E-05 & $-3,50961 \mathrm{E}-08$ \\
\hline & $\mu_{\mathrm{JT}, \mathrm{vapor}}$ & 7,44893E-03 & $-1,44523 \mathrm{E}-04$ & $1,13341 \mathrm{E}-06$ & $-4,46597 \mathrm{E}-09$ & $8,82211 \mathrm{E}-12$ & $-6,98269 \mathrm{E}-15$ \\
\hline \multirow{18}{*}{ R600a } & plíquido & $1,15706 \mathrm{E}+03$ & $-2,99754 \mathrm{E}+01$ & $2,86963 \mathrm{E}-01$ & $-1,25335 \mathrm{E}-03$ & 2,35059E-06 & $-1,16068 \mathrm{E}-09$ \\
\hline & p vapor & $1,15706 \mathrm{E}+03$ & $-2,99754 \mathrm{E}+01$ & 2,86963E-01 & $-1,25335 \mathrm{E}-03$ & 2,35059E-06 & $-1,16068 \mathrm{E}-09$ \\
\hline & ९líquido & $1,34445 \mathrm{E}+03$ & $-1,06220 \mathrm{E}+01$ & $7,53538 \mathrm{E}-02$ & $-2,93101 \mathrm{E}-04$ & 5,71205E-07 & $-4,54490 \mathrm{E}-10$ \\
\hline & $\rho_{\text {vapor }}$ & $-4,97442 \mathrm{E}+02$ & $9,49930 \mathrm{E}+00$ & $-7,23550 \mathrm{E}-02$ & 2,75930E-04 & $-5,31858 \mathrm{E}-07$ & 4,22703E-10 \\
\hline & $\mathrm{u}_{\text {líquido }}$ & $-4,72717 \mathrm{E}+05$ & 4,91097E+03 & $-2,54073 \mathrm{E}+01$ & 1,01379E-01 & $-1,92344 \mathrm{E}-04$ & $1,52118 \mathrm{E}-07$ \\
\hline & $\mathrm{h}_{\text {líquido }}$ & $-4,98305 \mathrm{E}+05$ & $5,40130 \mathrm{E}+03$ & $-2,91835 \mathrm{E}+01$ & 1,16118E-01 & $-2,21929 \mathrm{E}-04$ & 1,77110E-07 \\
\hline & Slíquido & $-3,48312 \mathrm{E}+03$ & $3,83606 \mathrm{E}+01$ & $-1,70376 \mathrm{E}-01$ & 5,17799E-04 & $-8,52265 \mathrm{E}-07$ & $5,97495 \mathrm{E}-10$ \\
\hline & cVlíquido & $-4,89179 \mathrm{E}+02$ & $3,18048 \mathrm{E}+01$ & $-2,42037 \mathrm{E}-01$ & $9,50410 \mathrm{E}-04$ & $-1,78334 \mathrm{E}-06$ & 1,31908E-09 \\
\hline & cplíquido & $-1,56964 \mathrm{E}+04$ & $3,32188 \mathrm{E}+02$ & $-2,53260 \mathrm{E}+00$ & 9,70264E-03 & $-1,85247 \mathrm{E}-05$ & 1,41955E-08 \\
\hline & SS líquido & $3,46702 \mathrm{E}+03$ & $-2,07330 \mathrm{E}+01$ & 1,01108E-01 & $-3,63990 \mathrm{E}-04$ & 6,96124E-07 & $-5,54508 \mathrm{E}-10$ \\
\hline & $\mu \mathrm{JT}$,liquido & $-3,30993 \mathrm{E}-05$ & $6,22887 \mathrm{E}-07$ & $-4,77866 \mathrm{E}-09$ & $1,83434 \mathrm{E}-11$ & $-3,51906 \mathrm{E}-14$ & $2,71048 \mathrm{E}-17$ \\
\hline & $\mathrm{u}_{\mathrm{vapor}}$ & $6,46563 \mathrm{E}+05$ & $-6,52101 \mathrm{E}+03$ & $5,41500 \mathrm{E}+01$ & $-1,98313 \mathrm{E}-01$ & $3,76726 \mathrm{E}-04$ & $-2,91741 \mathrm{E}-07$ \\
\hline & $\mathrm{h}_{\text {vapor }}$ & 7,36607E+05 & $-8,06331 \mathrm{E}+03$ & $6,65612 \mathrm{E}+01$ & $-2,43315 \mathrm{E}-01$ & 4,58233E-04 & $-3,53876 \mathrm{E}-07$ \\
\hline & Svapor & $9,93825 \mathrm{E}+03$ & $-1,08370 \mathrm{E}+02$ & $6,29739 \mathrm{E}-01$ & $-1,90332 \mathrm{E}-03$ & $3,01556 \mathrm{E}-06$ & $-1,98777 \mathrm{E}-09$ \\
\hline & $\mathrm{cV}_{\text {vapor }}$ & $2,38496 \mathrm{E}+03$ & $-3,38063 \mathrm{E}+01$ & 2,77146E-01 & $-1,04062 \mathrm{E}-03$ & 2,00113E-06 & $-1,53114 \mathrm{E}-09$ \\
\hline & $\mathrm{cp}_{\text {vapor }}$ & $-2,21295 \mathrm{E}+04$ & 4,38267E+02 & $-3,32623 \mathrm{E}+00$ & 1,26754E-02 & $-2,40715 \mathrm{E}-05$ & 1,83387E-08 \\
\hline & $\mathrm{SS}_{\text {vapor }}$ & $3,48570 \mathrm{E}+02$ & $-4,59087 \mathrm{E}+00$ & $3,87432 \mathrm{E}-02$ & -1,45397E-04 & 2,68531E-07 & $-2,09805 \mathrm{E}-10$ \\
\hline & $\mu_{\mathrm{JT}, \text { vapor }}$ & 1,99438E-03 & $-2,74859 \mathrm{E}-05$ & 1,57993E-07 & $-4,63886 \mathrm{E}-10$ & $6,88676 \mathrm{E}-13$ & $-4,10151 \mathrm{E}-16$ \\
\hline \multirow{18}{*}{ R1234YF } & plíquido & $-8,52604 \mathrm{E}+03$ & $1,46821 \mathrm{E}+02$ & $-9,92640 \mathrm{E}-01$ & 3,36479E-03 & $-6,12556 \mathrm{E}-06$ & $5,47476 \mathrm{E}-09$ \\
\hline & $\mathrm{p}_{\text {vapor }}$ & $-8,52604 \mathrm{E}+03$ & $1,46821 \mathrm{E}+02$ & $-9,92640 \mathrm{E}-01$ & 3,36479E-03 & $-6,12556 \mathrm{E}-06$ & 5,47476E-09 \\
\hline & ९líquido & $2,25499 \mathrm{E}+04$ & $-3,88612 \mathrm{E}+02$ & $2,87336 \mathrm{E}+00$ & $-1,06541 \mathrm{E}-02$ & 1,97073E-05 & $-1,45888 \mathrm{E}-08$ \\
\hline & $\rho_{\text {vapor }}$ & $-1,71651 \mathrm{E}+04$ & $3,21123 \mathrm{E}+02$ & $-2,39528 \mathrm{E}+00$ & 8,91077E-03 & $-1,65574 \mathrm{E}-05$ & 1,23323E-08 \\
\hline & $\mathrm{u}_{\text {líquido }}$ & $-1,66652 \mathrm{E}+06$ & $3,08786 \mathrm{E}+04$ & $-2,24985 \mathrm{E}+02$ & 8,40900E-01 & $-1,55805 \mathrm{E}-03$ & 1,15349E-06 \\
\hline & $\mathrm{h}_{\text {líquido }}$ & $-1,87780 \mathrm{E}+06$ & $3,48288 \mathrm{E}+04$ & $-2,54454 \mathrm{E}+02$ & 9,50677E-01 & $-1,76277 \mathrm{E}-03$ & 1,30710E-06 \\
\hline & Slíquido & $-5,75921 \mathrm{E}+03$ & $1,02651 \mathrm{E}+02$ & $-7,09325 \mathrm{E}-01$ & 2,59146E-03 & $-4,76070 \mathrm{E}-06$ & 3,50631E-09 \\
\hline & cVlíquido & $-2,40719 \mathrm{E}+04$ & 4,50083E+02 & $-3,28922 \mathrm{E}+00$ & $1,20515 \mathrm{E}-02$ & $-2,20205 \mathrm{E}-05$ & $1,60356 \mathrm{E}-08$ \\
\hline & cplíquido & $-3,26228 \mathrm{E}+05$ & $6,07748 \mathrm{E}+03$ & $-4,49898 \mathrm{E}+01$ & 1,65934E-01 & $-3,04858 \mathrm{E}-04$ & 2,23290E-07 \\
\hline & $\mathrm{SS}_{\text {líquido }}$ & $1,70871 \mathrm{E}+04$ & $-2,81002 \mathrm{E}+02$ & $2,00160 \mathrm{E}+00$ & $-7,26101 \mathrm{E}-03$ & 1,32032E-05 & $-9,62402 \mathrm{E}-09$ \\
\hline & $\mu_{\mathrm{JT} \text {,liquido }}$ & $-9,12546 \mathrm{E}-04$ & $1,69615 \mathrm{E}-05$ & $-1,25691 \mathrm{E}-07$ & $4,64000 \mathrm{E}-10$ & $-8,53485 \mathrm{E}-13$ & $6,26151 \mathrm{E}-16$ \\
\hline & $\mathrm{u}_{\text {vapor }}$ & $2,18123 \mathrm{E}+06$ & $-3,66064 \mathrm{E}+04$ & $2,76547 \mathrm{E}+02$ & $-1,02907 \mathrm{E}+00$ & $1,92025 \mathrm{E}-03$ & $-1,43862 \mathrm{E}-06$ \\
\hline & $h_{\text {vapor }}$ & $2,58895 \mathrm{E}+06$ & $-4,41573 \mathrm{E}+04$ & $3,33287 \mathrm{E}+02$ & $-1,23921 \mathrm{E}+00$ & 2,30847E-03 & $-1,72745 \mathrm{E}-06$ \\
\hline & Svapor & $1,05267 \mathrm{E}+04$ & $-1,51920 \mathrm{E}+02$ & $1,05212 \mathrm{E}+00$ & $-3,71096 \mathrm{E}-03$ & $6,65746 \mathrm{E}-06$ & $-4,84253 \mathrm{E}-09$ \\
\hline & $\mathrm{cV}_{\text {vapor }}$ & $4,58033 \mathrm{E}+03$ & $-6,77224 \mathrm{E}+01$ & 4,14491E-01 & $-1,10815 \mathrm{E}-03$ & 1,20773E-06 & $-2,26141 \mathrm{E}-10$ \\
\hline & $\mathrm{cp}_{\text {vapor }}$ & $-5,42802 \mathrm{E}+05$ & $1,00873 \mathrm{E}+04$ & $-7,46180 \mathrm{E}+01$ & 2,74969E-01 & $-5,04766 \mathrm{E}-04$ & $3,69443 \mathrm{E}-07$ \\
\hline & $\mathrm{SS}_{\text {vapor }}$ & 1,11277E+03 & $-1,94262 \mathrm{E}+01$ & 1,47882E-01 & $-5,49623 \mathrm{E}-04$ & $1,01665 \mathrm{E}-06$ & $-7,64780 \mathrm{E}-10$ \\
\hline & $\mu_{\mathrm{JT}, \text { vapor }}$ & 1,16094E-03 & $-1,37747 \mathrm{E}-05$ & 6,87591E-08 & $-1,78778 \mathrm{E}-10$ & $2,42590 \mathrm{E}-13$ & $-1,36601 \mathrm{E}-16$ \\
\hline
\end{tabular}




\begin{tabular}{|c|c|c|c|c|c|c|c|}
\hline \multirow{18}{*}{$\mathrm{R} 12$} & plíquido & $-2,04559 \mathrm{E}+03$ & $2,67154 \mathrm{E}+01$ & $-1,00412 \mathrm{E}-01$ & $2,57114 \mathrm{E}-05$ & 2,22566E-07 & $5,16485 \mathrm{E}-10$ \\
\hline & $\mathrm{p}_{\text {vapor }}$ & $-2,04559 \mathrm{E}+03$ & $2,67154 \mathrm{E}+01$ & $-1,00412 \mathrm{E}-01$ & $2,57114 \mathrm{E}-05$ & $2,22566 \mathrm{E}-07$ & $5,16485 \mathrm{E}-10$ \\
\hline & ९líquido & $6,55233 \mathrm{E}+03$ & $-8,76702 \mathrm{E}+01$ & 6,52373E-01 & $-2,49486 \mathrm{E}-03$ & 4,77033E-06 & $-3,67860 \mathrm{E}-09$ \\
\hline & $\rho_{\text {vapor }}$ & $-4,27543 \mathrm{E}+03$ & $8,18071 \mathrm{E}+01$ & $-6,23933 \mathrm{E}-01$ & 2,37483E-03 & $-4,52892 \mathrm{E}-06$ & $3,49375 \mathrm{E}-09$ \\
\hline & $\mathrm{u}_{\text {líquido }}$ & $-3,17017 \mathrm{E}+05$ & $6,46020 \mathrm{E}+03$ & $-4,37824 \mathrm{E}+01$ & $1,68111 \mathrm{E}-01$ & $-3,19851 \mathrm{E}-04$ & $2,45495 \mathrm{E}-07$ \\
\hline & hlíquido & $-3,61109 \mathrm{E}+05$ & 7,30324E+03 & $-5,02219 \mathrm{E}+01$ & $1,92760 \mathrm{E}-01$ & $-3,67453 \mathrm{E}-04$ & $2,83074 \mathrm{E}-07$ \\
\hline & Slíquido & $-1,63006 \mathrm{E}+03$ & $2,98156 \mathrm{E}+01$ & $-1,72604 \mathrm{E}-01$ & 5,94412E-04 & $-1,06881 \mathrm{E}-06$ & $7,89043 \mathrm{E}-10$ \\
\hline & cVlíquido & $-2,14639 \mathrm{E}+03$ & $5,16160 \mathrm{E}+01$ & $-4,03052 \mathrm{E}-01$ & 1,57859E-03 & $-3,06584 \mathrm{E}-06$ & 2,36731E-09 \\
\hline & cplíquido & $-3,86126 \mathrm{E}+04$ & $7,50708 \mathrm{E}+02$ & $-5,70386 \mathrm{E}+00$ & $2,16128 \mathrm{E}-02$ & $-4,08207 \mathrm{E}-05$ & $3,07993 \mathrm{E}-08$ \\
\hline & SS líquido & $3,31248 \mathrm{E}+03$ & $-3,24414 \mathrm{E}+01$ & $2,08787 \mathrm{E}-01$ & $-7,92124 \mathrm{E}-04$ & 1,54351E-06 & $-1,22710 \mathrm{E}-09$ \\
\hline & $\mu_{\mathrm{JT}}$,liquido & $-1,44835 \mathrm{E}-04$ & 2,75310E-06 & $-2,09501 \mathrm{E}-08$ & $7,95029 \mathrm{E}-11$ & $-1,50514 \mathrm{E}-13$ & $1,13952 \mathrm{E}-16$ \\
\hline & $\mathrm{u}_{\mathrm{vapor}}$ & $6,93567 \mathrm{E}+05$ & $-8,36039 \mathrm{E}+03$ & $6,53410 \mathrm{E}+01$ & $-2,44585 \mathrm{E}-01$ & $4,62465 \mathrm{E}-04$ & $-3,54695 \mathrm{E}-07$ \\
\hline & $\mathrm{h}_{\text {vapor }}$ & $8,01344 \mathrm{E}+05$ & $-1,03469 \mathrm{E}+04$ & $8,08751 \mathrm{E}+01$ & $-3,02761 \mathrm{E}-01$ & 5,71054E-04 & $-4,37421 \mathrm{E}-07$ \\
\hline & S vapor & $5,97144 \mathrm{E}+03$ & $-6,63074 \mathrm{E}+01$ & 4,15998E-01 & $-1,36002 \mathrm{E}-03$ & 2,30466E-06 & $-1,61162 \mathrm{E}-09$ \\
\hline & $\mathrm{CV}_{\text {vapor }}$ & $-2,48917 \mathrm{E}+03$ & $5,06315 \mathrm{E}+01$ & $-3,68213 \mathrm{E}-01$ & 1,37624E-03 & $-2,57260 \mathrm{E}-06$ & 1,92968E-09 \\
\hline & $\mathrm{cp}_{\text {vapor }}$ & $-6,04430 \mathrm{E}+04$ & $1,15408 \mathrm{E}+03$ & $-8,72868 \mathrm{E}+00$ & 3,29337E-02 & $-6,19532 \mathrm{E}-05$ & 4,65516E-08 \\
\hline & $\mathrm{SS}_{\text {vapor }}$ & $3,30429 \mathrm{E}+02$ & $-4,89038 \mathrm{E}+00$ & 4,01688E-02 & $-1,52004 \mathrm{E}-04$ & 2,83245E-07 & $-2,19578 \mathrm{E}-10$ \\
\hline & $\mu_{\mathrm{JT}, \mathrm{vapor}}$ & 2,22926E-03 & $-3,27476 \mathrm{E}-05$ & 1,99804E-07 & $-6,21222 \mathrm{E}-10$ & $9,76570 \mathrm{E}-13$ & $-6,17323 \mathrm{E}-16$ \\
\hline \multirow{18}{*}{$\mathrm{R} 134 \mathrm{a}$} & plíquido & $-3,43265 E+03$ & $5,11464 \mathrm{E}+01$ & $-2,85189 \mathrm{E}-01$ & 8,18183E-04 & $-1,73722 \mathrm{E}-06$ & 2,64987E-09 \\
\hline & $\mathrm{p}_{\text {vapor }}$ & $-3,43265 E+03$ & $5,11464 \mathrm{E}+01$ & $-2,85189 \mathrm{E}-01$ & 8,18183E-04 & $-1,73722 \mathrm{E}-06$ & 2,64987E-09 \\
\hline & ९líquido & 1,04147E+04 & $-1,63089 \mathrm{E}+02$ & $1,22568 \mathrm{E}+00$ & $-4,66984 \mathrm{E}-03$ & 8,89053E-06 & $-6,80142 \mathrm{E}-09$ \\
\hline & $\rho_{\text {vapor }}$ & $-8,63806 \mathrm{E}+03$ & $1,64641 \mathrm{E}+02$ & $-1,25100 \mathrm{E}+00$ & 4,74263E-03 & $-8,99268 \mathrm{E}-06$ & 6,85587E-09 \\
\hline & ulíquido & $-8,64043 \mathrm{E}+05$ & 1,53934E+04 & $-1,09384 \mathrm{E}+02$ & 4,18292E-01 & $-7,95864 \mathrm{E}-04$ & $6,08980 \mathrm{E}-07$ \\
\hline & $\mathrm{h}_{\text {líquido }}$ & $-9,70622 \mathrm{E}+05$ & $1,74282 \mathrm{E}+04$ & $-1,24898 \mathrm{E}+02$ & 4,77468E-01 & $-9,09249 \mathrm{E}-04$ & 6,96892E-07 \\
\hline & Slíquido & $-3,71486 \mathrm{E}+03$ & $6,05249 \mathrm{E}+01$ & $-3,81733 \mathrm{E}-01$ & 1,36030E-03 & $-2,49566 \mathrm{E}-06$ & 1,86367E-09 \\
\hline & cVlíquido & $-4,53068 \mathrm{E}+03$ & $1,00472 \mathrm{E}+02$ & $-7,65262 \mathrm{E}-01$ & $2,92056 \mathrm{E}-03$ & $-5,53665 \mathrm{E}-06$ & 4,18471E-09 \\
\hline & cplíquido & $-1,30528 \mathrm{E}+05$ & $2,49321 \mathrm{E}+03$ & $-1,88075 \mathrm{E}+01$ & 7,06739E-02 & $-1,32299 \mathrm{E}-04$ & $9,88059 \mathrm{E}-08$ \\
\hline & SSlíquido & $5,89487 \mathrm{E}+03$ & $-7,64623 \mathrm{E}+01$ & $5,18680 \mathrm{E}-01$ & $-1,88782 \mathrm{E}-03$ & 3,46397E-06 & $-2,56243 \mathrm{E}-09$ \\
\hline & $\mu_{\mathrm{JT}}$,liquido & $-2,76358 \mathrm{E}-04$ & $5,23899 \mathrm{E}-06$ & $-3,96297 \mathrm{E}-08$ & $1,49355 \mathrm{E}-10$ & $-2,80556 \mathrm{E}-13$ & $2,10392 \mathrm{E}-16$ \\
\hline & $\mathrm{u}_{\mathrm{vapor}}$ & $1,52579 \mathrm{E}+06$ & $-2,38868 \mathrm{E}+04$ & $1,83412 \mathrm{E}+02$ & $-6,88302 \mathrm{E}-01$ & $1,29539 \mathrm{E}-03$ & $-9,80487 \mathrm{E}-07$ \\
\hline & $\mathrm{h}_{\text {vapor }}$ & $1,77694 \mathrm{E}+06$ & $-2,85863 \mathrm{E}+04$ & $2,19545 \mathrm{E}+02$ & $-8,23902 \mathrm{E}-01$ & 1,54913E-03 & $-1,17261 \mathrm{E}-06$ \\
\hline & S Sapor & $9,86280 \mathrm{E}+03$ & $-1,30415 \mathrm{E}+02$ & 8,67298E-01 & $-2,97614 \mathrm{E}-03$ & 5,23527E-06 & $-3,75697 \mathrm{E}-09$ \\
\hline & $\mathrm{CV}_{\text {vapor }}$ & $-7,33042 \mathrm{E}+03$ & $1,45762 \mathrm{E}+02$ & $-1,09602 \mathrm{E}+00$ & $4,14758 \mathrm{E}-03$ & -7,78369E-06 & 5,82287E-09 \\
\hline & $\mathrm{cp}_{\text {vapor }}$ & $-2,23301 \mathrm{E}+05$ & $4,23454 \mathrm{E}+03$ & $-3,19058 \mathrm{E}+01$ & 1,19720E-01 & $-2,23690 \mathrm{E}-04$ & $1,66650 \mathrm{E}-07$ \\
\hline & $\mathrm{SS}_{\text {vapor }}$ & $6,24437 \mathrm{E}+02$ & $-1,04297 \mathrm{E}+01$ & 8,27473E-02 & $-3,14190 \mathrm{E}-04$ & 5,92712E-07 & $-4,59571 \mathrm{E}-10$ \\
\hline & $\mu_{\mathrm{JT}, \mathrm{vapor}}$ & 8,49516E-03 & -1,36069E-04 & 8,85981E-07 & $-2,90778 \mathrm{E}-09$ & $4,79060 \mathrm{E}-12$ & $-3,16113 \mathrm{E}-15$ \\
\hline \multirow{18}{*}{ R22 } & plíquido & $-8,97205 E+03$ & $1,52395 \mathrm{E}+02$ & $-9,99374 \mathrm{E}-01$ & $3,22391 \mathrm{E}-03$ & $-5,62289 \mathrm{E}-06$ & $5,26502 \mathrm{E}-09$ \\
\hline & $\mathrm{p}_{\text {vapor }}$ & $-8,97205 E+03$ & $1,52395 \mathrm{E}+02$ & $-9,99374 \mathrm{E}-01$ & 3,22391E-03 & $-5,62289 \mathrm{E}-06$ & $5,26502 \mathrm{E}-09$ \\
\hline & ९líquido & 1,61852E+04 & $-2,72497 \mathrm{E}+02$ & $2,04852 \mathrm{E}+00$ & $-7,74375 \mathrm{E}-03$ & $1,45962 \mathrm{E}-05$ & $-1,10156 \mathrm{E}-08$ \\
\hline & $\rho_{\text {vapor }}$ & $-1,27277 \mathrm{E}+04$ & $2,42224 \mathrm{E}+02$ & $-1,83670 \mathrm{E}+00$ & 6,94188E-03 & $-1,31019 \mathrm{E}-05$ & $9,91945 \mathrm{E}-09$ \\
\hline & ulíquido & $-1,54512 \mathrm{E}+06$ & $2,86226 \mathrm{E}+04$ & $-2,08031 \mathrm{E}+02$ & $7,81090 \mathrm{E}-01$ & $-1,46217 \mathrm{E}-03$ & $1,09753 \mathrm{E}-06$ \\
\hline & $\mathrm{h}_{\text {líquido }}$ & $-1,73241 \mathrm{E}+06$ & $3,21853 \mathrm{E}+04$ & $-2,35058 \mathrm{E}+02$ & $8,83455 \mathrm{E}-01$ & $-1,65635 \mathrm{E}-03$ & $1,24601 \mathrm{E}-06$ \\
\hline & Slíquido & $-5,82322 \mathrm{E}+03$ & 1,02931E+02 & $-7,02276 \mathrm{E}-01$ & 2,54289E-03 & $-4,66413 \mathrm{E}-06$ & $3,44945 \mathrm{E}-09$ \\
\hline & cVlíquido & $-4,77198 \mathrm{E}+03$ & $1,07854 \mathrm{E}+02$ & $-8,40741 \mathrm{E}-01$ & $3,21627 \mathrm{E}-03$ & $-6,05671 \mathrm{E}-06$ & 4,52481E-09 \\
\hline & cplíquido & $-2,76494 \mathrm{E}+05$ & $5,24967 \mathrm{E}+03$ & $-3,95241 \mathrm{E}+01$ & 1,48071E-01 & $-2,76088 \mathrm{E}-04$ & 2,05119E-07 \\
\hline & SS $_{\text {líquido }}$ & 4,22663E+03 & $-5,00659 \mathrm{E}+01$ & 3,59319E-01 & $-1,42256 \mathrm{E}-03$ & 2,82214E-06 & $-2,24843 \mathrm{E}-09$ \\
\hline & $\mu_{\mathrm{JT} \text {, liquido }}$ & $-4,26330 \mathrm{E}-04$ & $8,08477 \mathrm{E}-06$ & $-6,11319 \mathrm{E}-08$ & $2,30199 \mathrm{E}-10$ & $-4,31846 \mathrm{E}-13$ & $3,23206 \mathrm{E}-16$ \\
\hline & $\mathrm{u}_{\mathrm{vapor}}$ & $2,10695 \mathrm{E}+06$ & $-3,44486 \mathrm{E}+04$ & $2,63689 \mathrm{E}+02$ & $-9,94467 \mathrm{E}-01$ & $1,87368 \mathrm{E}-03$ & $-1,41592 \mathrm{E}-06$ \\
\hline & $\mathrm{h}_{\text {vapor }}$ & $2,49926 \mathrm{E}+06$ & $-4,18353 \mathrm{E}+04$ & $3,20415 \mathrm{E}+02$ & $-1,20809 \mathrm{E}+00$ & 2,27429E-03 & $-1,71849 \mathrm{E}-06$ \\
\hline & S vapor & $1,18335 \mathrm{E}+04$ & $-1,64290 \mathrm{E}+02$ & $1,12070 \mathrm{E}+00$ & $-3,94337 \mathrm{E}-03$ & $7,07216 \mathrm{E}-06$ & $-5,14710 \mathrm{E}-09$ \\
\hline & $\mathrm{cv}_{\text {vapor }}$ & $-9,11689 \mathrm{E}+03$ & $1,79226 \mathrm{E}+02$ & $-1,35478 \mathrm{E}+00$ & 5,12107E-03 & $-9,60732 \mathrm{E}-06$ & 7,18786E-09 \\
\hline & $\mathrm{cp}_{\text {vapor }}$ & $-4,18955 \mathrm{E}+05$ & $7,91518 \mathrm{E}+03$ & $-5,94662 \mathrm{E}+01$ & 2,22364E-01 & $-4,13902 \mathrm{E}-04$ & $3,07008 \mathrm{E}-07$ \\
\hline & $\mathrm{SS}_{\text {vapor }}$ & $5,26969 \mathrm{E}+02$ & $-8,45010 \mathrm{E}+00$ & 6,87694E-02 & $-2,63592 \mathrm{E}-04$ & 4,98462E-07 & $-3,87560 \mathrm{E}-10$ \\
\hline & $\mu_{\mathrm{JT}, \mathrm{vapor}}$ & 2,54817E-03 & $-3,78170$ E-05 & $2,35185 \mathrm{E}-07$ & $-7,51694 \mathrm{E}-10$ & $1,22339 \mathrm{E}-12$ & $-8,06109 \mathrm{E}-16$ \\
\hline
\end{tabular}




\begin{tabular}{|c|c|c|c|c|c|c|c|}
\hline \multirow{18}{*}{$\mathrm{R} 32$} & plíquido & $-3,33150 \mathrm{E}+04$ & $6,17188 \mathrm{E}+02$ & $-4,53381 \mathrm{E}+00$ & $1,66631 \mathrm{E}-02$ & $-3,14715 \mathrm{E}-05$ & 2,59227E-08 \\
\hline & $\mathrm{p}_{\text {vapor }}$ & $-3,33150 \mathrm{E}+04$ & $6,17188 \mathrm{E}+02$ & $-4,53381 \mathrm{E}+00$ & $1,66631 \mathrm{E}-02$ & $-3,14715 \mathrm{E}-05$ & 2,59227E-08 \\
\hline & ९líquido & $5,37820 \mathrm{E}+04$ & $-9,92772 \mathrm{E}+02$ & $7,50890 \mathrm{E}+00$ & $-2,83496 \mathrm{E}-02$ & 5,33202E-05 & $-4,00220 \mathrm{E}-08$ \\
\hline & $\rho_{\text {vapor }}$ & $-4,90302 \mathrm{E}+04$ & $9,32546 \mathrm{E}+02$ & $-7,06379 \mathrm{E}+00$ & $2,66452 \mathrm{E}-02$ & $-5,00831 \mathrm{E}-05$ & 3,75767E-08 \\
\hline & $\mathrm{u}_{\text {líquido }}$ & $-9,15682 \mathrm{E}+06$ & $1,71376 \mathrm{E}+05$ & $-1,28642 \mathrm{E}+03$ & $4,85177 \mathrm{E}+00$ & $-9,11749 \mathrm{E}-03$ & 6,84055E-06 \\
\hline & $\mathrm{h}_{\text {líquido }}$ & $-1,04025 \mathrm{E}+07$ & $1,95078 \mathrm{E}+05$ & $-1,46607 \mathrm{E}+03$ & $5,53024 \mathrm{E}+00$ & $-1,03954 \mathrm{E}-02$ & $7,80296 \mathrm{E}-06$ \\
\hline & Slíquido & $-3,05672 \mathrm{E}+04$ & $5,61151 \mathrm{E}+02$ & $-4,13902 \mathrm{E}+00$ & $1,54656 \mathrm{E}-02$ & $-2,89110 \mathrm{E}-05$ & 2,16088E-08 \\
\hline & cVlíquido & $-8,58632 \mathrm{E}+04$ & $1,65722 \mathrm{E}+03$ & $-1,25767 \mathrm{E}+01$ & 4,74498E-02 & $-8,90662 \mathrm{E}-05$ & 6,66045E-08 \\
\hline & cplíquido & $-4,48473 \mathrm{E}+06$ & $8,46013 \mathrm{E}+04$ & $-6,34774 \mathrm{E}+02$ & $2,36888 \mathrm{E}+00$ & $-4,39733 \mathrm{E}-03$ & $3,24885 \mathrm{E}-06$ \\
\hline & SS $_{\text {líquido }}$ & 1,30403E+04 & $-2,15760 \mathrm{E}+02$ & $1,62028 \mathrm{E}+00$ & $-6,22722 \mathrm{E}-03$ & $1,19778 \mathrm{E}-05$ & $-9,25092 \mathrm{E}-09$ \\
\hline & $\mu_{\mathrm{JT}}$,liquido & $-1,76231 \mathrm{E}-03$ & $3,34406 \mathrm{E}-05$ & $-2,52685 \mathrm{E}-07$ & $9,50351 \mathrm{E}-10$ & $-1,77942 \mathrm{E}-12$ & $1,32751 \mathrm{E}-15$ \\
\hline & $\mathrm{u}_{\mathrm{vapor}}$ & $1,29649 \mathrm{E}+07$ & $-2,39680 \mathrm{E}+05$ & $1,82212 \mathrm{E}+03$ & $-6,88393 \mathrm{E}+00$ & $1,29582 \mathrm{E}-02$ & $-9,73910 \mathrm{E}-06$ \\
\hline & $\mathrm{h}_{\text {vapor }}$ & $1,53918 \mathrm{E}+07$ & $-2,85736 \mathrm{E}+05$ & $2,17242 \mathrm{E}+03$ & $-8,20487 \mathrm{E}+00$ & 1,54382E-02 & $-1,16008 \mathrm{E}-05$ \\
\hline & S vapor & $5,03634 \mathrm{E}+04$ & $-8,69335 \mathrm{E}+02$ & $6,38658 \mathrm{E}+00$ & $-2,36613 \mathrm{E}-02$ & 4,39591E-05 & $-3,27201 E-08$ \\
\hline & $\mathrm{cV}_{\text {vapor }}$ & $-5,04222 \mathrm{E}+04$ & $9,72776 \mathrm{E}+02$ & $-7,43418 \mathrm{E}+00$ & 2,83787E-02 & $-5,39438 \mathrm{E}-05$ & 4,08844E-08 \\
\hline & $\mathrm{cp}_{\text {vapor }}$ & $-7,68974 \mathrm{E}+06$ & $1,45016 \mathrm{E}+05$ & $-1,08806 \mathrm{E}+03$ & $4,06056 \mathrm{E}+00$ & $-7,53787 \mathrm{E}-03$ & 5,56937E-06 \\
\hline & $\mathrm{SS}_{\text {vapor }}$ & 4,21084E+03 & $-7,81256 \mathrm{E}+01$ & 5,98880E-01 & $-2,26588 \mathrm{E}-03$ & 4,25891E-06 & $-3,20480$ E-09 \\
\hline & $\mu_{\mathrm{JT}, \mathrm{vapor}}$ & 8,31391E-03 & $-1,29283 \mathrm{E}-04$ & 8,23647E-07 & $-2,66824 \mathrm{E}-09$ & 4,37867E-12 & $-2,90481 \mathrm{E}-15$ \\
\hline \multirow{18}{*}{$\mathrm{R} 404 \mathrm{a}$} & plíquido & $-2,36577 \mathrm{E}+04$ & $4,41392 \mathrm{E}+02$ & $-3,26202 \mathrm{E}+00$ & 1,20385E-02 & $-2,27670 \mathrm{E}-05$ & 1,87290E-08 \\
\hline & $\mathrm{p}_{\text {vapor }}$ & $-2,65271 \mathrm{E}+04$ & $4,96120 \mathrm{E}+02$ & $-3,67868 \mathrm{E}+00$ & $1,36246 \mathrm{E}-02$ & $-2,57932 \mathrm{E}-05$ & 2,10434E-08 \\
\hline & ९líquido & $6,60450 \mathrm{E}+04$ & $-1,23233 \mathrm{E}+03$ & $9,39740 \mathrm{E}+00$ & $-3,57833 \mathrm{E}-02$ & $6,79224 \mathrm{E}-05$ & $-5,14811 \mathrm{E}-08$ \\
\hline & $\rho_{\text {vapor }}$ & $-6,56075 E+04$ & $1,25788 \mathrm{E}+03$ & $-9,60795 \mathrm{E}+00$ & $3,65568 \mathrm{E}-02$ & $-6,93286 \mathrm{E}-05$ & 5,24919E-08 \\
\hline & ulíquido & $-5,61321 \mathrm{E}+06$ & $1,06413 \mathrm{E}+05$ & $-8,04540 \mathrm{E}+02$ & $3,06292 \mathrm{E}+00$ & $-5,80981 \mathrm{E}-03$ & 4,40178E-06 \\
\hline & $\mathrm{h}_{\text {líquido }}$ & $-6,40603 E+06$ & $1,21623 \mathrm{E}+05$ & $-9,20810 \mathrm{E}+02$ & $3,50591 \mathrm{E}+00$ & $-6,65186 \mathrm{E}-03$ & 5,04186E-06 \\
\hline & Slíquido & $-1,92256 \mathrm{E}+04$ & $3,57611 \mathrm{E}+02$ & $-2,64907 \mathrm{E}+00$ & 9,97899E-03 & $-1,88205 \mathrm{E}-05$ & 1,42002E-08 \\
\hline & cVlíquido & $-5,67916 \mathrm{E}+04$ & 1,09657E+03 & $-8,33286 \mathrm{E}+00$ & $3,15601 \mathrm{E}-02$ & $-5,95277 \mathrm{E}-05$ & 4,47571E-08 \\
\hline & cplíquido & $-2,95602 \mathrm{E}+06$ & $5,62150 \mathrm{E}+04$ & $-4,25352 \mathrm{E}+02$ & $1,60140 \mathrm{E}+00$ & $-3,00009 \mathrm{E}-03$ & $2,23780 \mathrm{E}-06$ \\
\hline & SSlíquido & $2,13507 \mathrm{E}+04$ & $-3,67733 \mathrm{E}+02$ & $2,69343 \mathrm{E}+00$ & $-9,99235 \mathrm{E}-03$ & $1,85460 \mathrm{E}-05$ & $-1,37875 \mathrm{E}-08$ \\
\hline & $\mu_{\mathrm{JT}}$,liquido & $-3,37455 \mathrm{E}-03$ & $6,44406 \mathrm{E}-05$ & $-4,90081 \mathrm{E}-07$ & 1,85544E-09 & $-3,49755 \mathrm{E}-12$ & $2,62690 \mathrm{E}-15$ \\
\hline & $\mathrm{u}_{\mathrm{vapor}}$ & $8,61942 \mathrm{E}+06$ & $-1,60721 \mathrm{E}+05$ & $1,23062 \mathrm{E}+03$ & $-4,68054 \mathrm{E}+00$ & $8,87889 \mathrm{E}-03$ & $-6,72654 \mathrm{E}-06$ \\
\hline & $\mathrm{h}_{\text {vapor }}$ & $1,01687 \mathrm{E}+07$ & $-1,90380 \mathrm{E}+05$ & $1,45803 \mathrm{E}+03$ & $-5,54601 \mathrm{E}+00$ & 1,05199E-02 & $-7,97090 \mathrm{E}-06$ \\
\hline & S Sapor & $3,33637 \mathrm{E}+04$ & $-5,87283 \mathrm{E}+02$ & $4,37472 \mathrm{E}+00$ & $-1,63614 \mathrm{E}-02$ & $3,06652 \mathrm{E}-05$ & $-2,30206 \mathrm{E}-08$ \\
\hline & $\mathrm{CV}_{\text {vapor }}$ & $-5,17535 \mathrm{E}+04$ & $9,95316 \mathrm{E}+02$ & $-7,57053 \mathrm{E}+00$ & 2,87454E-02 & $-5,43764 \mathrm{E}-05$ & 4,10416E-08 \\
\hline & $\mathrm{cp}_{\text {vapor }}$ & $-4,93054 \mathrm{E}+06$ & $9,37362 \mathrm{E}+04$ & $-7,09251 \mathrm{E}+02$ & $2,67026 \mathrm{E}+00$ & $-5,00257 \mathrm{E}-03$ & 3,73144E-06 \\
\hline & $\mathrm{SS}_{\text {vapor }}$ & $1,76078 \mathrm{E}+03$ & $-3,26213 \mathrm{E}+01$ & 2,56039E-01 & $-9,88324 \mathrm{E}-04$ & 1,89996E-06 & $-1,47731 E-09$ \\
\hline & $\mu_{\mathrm{JT}, \mathrm{vapor}}$ & 9,67987E-03 & -1,63094E-04 & 1,11396E-06 & $-3,83258 \mathrm{E}-09$ & $6,62292 \mathrm{E}-12$ & $-4,58941 \mathrm{E}-15$ \\
\hline \multirow{18}{*}{$\mathrm{R} 407 \mathrm{c}$} & plíquido & $-2,54505 \mathrm{E}+03$ & $3,07189 \mathrm{E}+01$ & $-8,19288 \mathrm{E}-02$ & $-2,05399 \mathrm{E}-04$ & $6,58775 \mathrm{E}-07$ & $8,90252 \mathrm{E}-10$ \\
\hline & $\mathrm{p}_{\text {vapor }}$ & $-4,23626 \mathrm{E}+04$ & $7,85536 \mathrm{E}+02$ & $-5,79650 \mathrm{E}+00$ & $2,14230 \mathrm{E}-02$ & $-4,02994 \mathrm{E}-05$ & 3,18798E-08 \\
\hline & ९líquido & 4,33196Е+04 & $-7,83033 \mathrm{E}+02$ & $5,87083 \mathrm{E}+00$ & $-2,19950 \mathrm{E}-02$ & $4,10596 \mathrm{E}-05$ & $-3,06067 \mathrm{E}-08$ \\
\hline & $\rho_{\text {vapor }}$ & $-3,59926 \mathrm{E}+04$ & $6,80350 \mathrm{E}+02$ & $-5,12079 \mathrm{E}+00$ & 1,91929E-02 & $-3,58541 \mathrm{E}-05$ & 2,67557E-08 \\
\hline & ulíquido & $-4,13828 \mathrm{E}+06$ & $7,66076 \mathrm{E}+04$ & $-5,66647 \mathrm{E}+02$ & $2,12107 \mathrm{E}+00$ & $-3,95522 \mathrm{E}-03$ & $2,94700 \mathrm{E}-06$ \\
\hline & $\mathrm{h}_{\text {líquido }}$ & $-4,69857 \mathrm{E}+06$ & $8,71988 \mathrm{E}+04$ & $-6,46390 \mathrm{E}+02$ & $2,42022 \mathrm{E}+00$ & $-4,51521 \mathrm{E}-03$ & 3,36661E-06 \\
\hline & Slíquido & $-1,41763 \mathrm{E}+04$ & $2,55042 \mathrm{E}+02$ & $-1,82913 \mathrm{E}+00$ & 6,73481E-03 & $-1,24451 \mathrm{E}-05$ & 9,21279E-09 \\
\hline & cVlíquido & $-4,21282 \mathrm{E}+04$ & $8,09496 \mathrm{E}+02$ & $-6,07713 \mathrm{E}+00$ & 2,27038E-02 & $-4,21943 \mathrm{E}-05$ & $3,12365 \mathrm{E}-08$ \\
\hline & cplíquido & $-1,64608 \mathrm{E}+06$ & $3,08580 \mathrm{E}+04$ & $-2,29924 \mathrm{E}+02$ & 8,51863E-01 & $-1,56955 \mathrm{E}-03$ & 1,15081E-06 \\
\hline & SS $_{\text {líquido }}$ & $1,44400 \mathrm{E}+04$ & $-2,35052 \mathrm{E}+02$ & 1,69317E+00 & $-6,23262 \mathrm{E}-03$ & $1,14893 \mathrm{E}-05$ & $-8,49135 \mathrm{E}-09$ \\
\hline & $\mu_{\mathrm{JT} \text {, liquido }}$ & $-1,66921 \mathrm{E}-03$ & $3,14057 \mathrm{E}-05$ & $-2,35203 \mathrm{E}-07$ & $8,76371 \mathrm{E}-10$ & $-1,62495 \mathrm{E}-12$ & $1,19999 \mathrm{E}-15$ \\
\hline & $\mathrm{u}_{\mathrm{vapor}}$ & $5,64731 \mathrm{E}+06$ & $-1,01598 \mathrm{E}+05$ & $7,68593 \mathrm{E}+02$ & $-2,88136 \mathrm{E}+00$ & $5,38586 \mathrm{E}-03$ & $-4,02173 \mathrm{E}-06$ \\
\hline & $\mathrm{h}_{\text {vapor }}$ & $6,70934 \mathrm{E}+06$ & $-1,21601 \mathrm{E}+05$ & $9,19924 \mathrm{E}+02$ & $-3,44797 \mathrm{E}+00$ & $6,44230 \mathrm{E}-03$ & $-4,81025 \mathrm{E}-06$ \\
\hline & S vapor & $2,40700 \mathrm{E}+04$ & $-3,92220 \mathrm{E}+02$ & $2,81520 \mathrm{E}+00$ & $-1,02257 \mathrm{E}-02$ & $1,86965 \mathrm{E}-05$ & $-1,37336 \mathrm{E}-08$ \\
\hline & $\mathrm{CV}_{\text {vapor }}$ & $-3,06529 \mathrm{E}+04$ & $5,88627 \mathrm{E}+02$ & $-4,44879 \mathrm{E}+00$ & 1,67883E-02 & $-3,15082 \mathrm{E}-05$ & $2,35586 \mathrm{E}-08$ \\
\hline & $\mathrm{cp}_{\text {vapor }}$ & $-1,65955 E+06$ & $3,11462 \mathrm{E}+04$ & $-2,32487 \mathrm{E}+02$ & $8,63100 \mathrm{E}-01$ & $-1,59377 \mathrm{E}-03$ & 1,17141E-06 \\
\hline & $\mathrm{SS}_{\text {vapor }}$ & $1,82729 \mathrm{E}+03$ & $-3,30933 \mathrm{E}+01$ & 2,54263E-01 & $-9,58743 \mathrm{E}-04$ & 1,79745E-06 & $-1,35880 \mathrm{E}-09$ \\
\hline & $\mu_{\mathrm{JT}, \mathrm{vapor}}$ & 5,72532E-03 & $-8,76176 \mathrm{E}-05$ & 5,51305E-07 & -1,76664E-09 & $2,86884 \mathrm{E}-12$ & $-1,88188 \mathrm{E}-15$ \\
\hline
\end{tabular}




\begin{tabular}{|c|c|c|c|c|c|c|c|}
\hline \multirow{18}{*}{$\mathrm{R} 410 \mathrm{a}$} & plíquido & $-2,67556 \mathrm{E}+04$ & 4,98447E+02 & $-3,67972 \mathrm{E}+00$ & $1,36022 \mathrm{E}-02$ & $-2,59718 \mathrm{E}-05$ & 2,19134E-08 \\
\hline & $\mathrm{p}_{\text {vapor }}$ & $-3,39619 \mathrm{E}+04$ & $6,36590 \mathrm{E}+02$ & $-4,73440 \mathrm{E}+00$ & 1,76106E-02 & $-3,35545 \mathrm{E}-05$ & 2,76213E-08 \\
\hline & ९líquido & 7,93057E+04 & $-1,48483 \mathrm{E}+03$ & $1,13164 \mathrm{E}+01$ & $-4,30417 \mathrm{E}-02$ & $8,15819 \mathrm{E}-05$ & $-6,17178 \mathrm{E}-08$ \\
\hline & $\rho_{\text {vapor }}$ & $-6,88599 \mathrm{E}+04$ & $1,32156 \mathrm{E}+03$ & $-1,01049 \mathrm{E}+01$ & 3,84897E-02 & $-7,30767 \mathrm{E}-05$ & 5,53918E-08 \\
\hline & $\mathrm{u}_{\text {líquido }}$ & $-8,58234 \mathrm{E}+06$ & $1,62351 \mathrm{E}+05$ & $-1,22754 \mathrm{E}+03$ & $4,66086 \mathrm{E}+00$ & $-8,82014 \mathrm{E}-03$ & 6,66535E-06 \\
\hline & hlíquido & $-9,72159 \mathrm{E}+06$ & $1,84211 \mathrm{E}+05$ & $-1,39468 \mathrm{E}+03$ & $5,29776 \mathrm{E}+00$ & $-1,00308 \mathrm{E}-02$ & $7,58543 \mathrm{E}-06$ \\
\hline & Slíquido & $-2,92246 \mathrm{E}+04$ & $5,44126 \mathrm{E}+02$ & $-4,04981 \mathrm{E}+00$ & 1,52447E-02 & $-2,87036 \mathrm{E}-05$ & $2,16085 \mathrm{E}-08$ \\
\hline & cVlíquido & $-1,16296 \mathrm{E}+05$ & $2,24931 \mathrm{E}+03$ & $-1,71896 \mathrm{E}+01$ & $6,53618 \mathrm{E}-02$ & $-1,23693 \mathrm{E}-04$ & $9,32660 \mathrm{E}-08$ \\
\hline & cplíquido & $-4,96404 \mathrm{E}+06$ & $9,44135 \mathrm{E}+04$ & $-7,14527 \mathrm{E}+02$ & $2,69049 \mathrm{E}+00$ & $-5,04085 \mathrm{E}-03$ & $3,76002 \mathrm{E}-06$ \\
\hline & SS $_{\text {líquido }}$ & 1,83091E+04 & $-3,20628 \mathrm{E}+02$ & $2,42512 \mathrm{E}+00$ & $-9,29668 \mathrm{E}-03$ & $1,78066 \mathrm{E}-05$ & $-1,36565 \mathrm{E}-08$ \\
\hline & $\mu_{\mathrm{JT}}$,liquido & $-2,99754 \mathrm{E}-03$ & $5,73018 \mathrm{E}-05$ & $-4,36263 \mathrm{E}-07$ & 1,65352E-09 & $-3,12050 \mathrm{E}-12$ & $2,34646 \mathrm{E}-15$ \\
\hline & $\mathrm{u}_{\mathrm{vapor}}$ & $1,17053 \mathrm{E}+07$ & $-2,19298 \mathrm{E}+05$ & $1,68291 \mathrm{E}+03$ & $-6,42019 \mathrm{E}+00$ & 1,22099E-02 & $-9,27193 \mathrm{E}-06$ \\
\hline & $\mathrm{h}_{\text {vapor }}$ & $1,38215 \mathrm{E}+07$ & $-2,59867 \mathrm{E}+05$ & $1,99440 \mathrm{E}+03$ & $-7,60747 \mathrm{E}+00$ & 1,44644E-02 & $-1,09836 \mathrm{E}-05$ \\
\hline & S vapor & 4,46692E+04 & $-7,90666 \mathrm{E}+02$ & $5,90502 \mathrm{E}+00$ & $-2,21760 \mathrm{E}-02$ & 4,17268E-05 & $-3,14394 \mathrm{E}-08$ \\
\hline & $\mathrm{cV}_{\text {vapor }}$ & $-8,34880 \mathrm{E}+04$ & $1,60880 \mathrm{E}+03$ & $-1,23108 \mathrm{E}+01$ & 4,70187E-02 & $-8,94810 \mathrm{E}-05$ & 6,79189E-08 \\
\hline & $\mathrm{cp}_{\text {vapor }}$ & $-7,79561 E+06$ & $1,48343 \mathrm{E}+05$ & $-1,12358 \mathrm{E}+03$ & $4,23462 \mathrm{E}+00$ & $-7,94203 \mathrm{E}-03$ & $5,93065 \mathrm{E}-06$ \\
\hline & $\mathrm{SS}_{\text {vapor }}$ & 4,08501E+03 & $-7,66658 \mathrm{E}+01$ & 5,91127E-01 & $-2,25368 \mathrm{E}-03$ & 4,27350E-06 & $-3,24762 \mathrm{E}-09$ \\
\hline & $\mu_{\mathrm{JT}, \mathrm{vapor}}$ & $8,45090 \mathrm{E}-03$ & $-1,35776 \mathrm{E}-04$ & 8,93091E-07 & $-2,98565 \mathrm{E}-09$ & $5,05363 \mathrm{E}-12$ & $-3,45545 \mathrm{E}-15$ \\
\hline \multirow{18}{*}{$\mathrm{R} 507 \mathrm{a}$} & plíquido & $-2,75132 \mathrm{E}+04$ & $5,16178 \mathrm{E}+02$ & $-3,83917 \mathrm{E}+00$ & $1,42550 \mathrm{E}-02$ & $-2,70131 \mathrm{E}-05$ & 2,19967E-08 \\
\hline & $\mathrm{p}_{\text {vapor }}$ & $-2,93806 \mathrm{E}+04$ & $5,51980 \mathrm{E}+02$ & $-4,11261 \mathrm{E}+00$ & $1,52947 \mathrm{E}-02$ & $-2,89806 \mathrm{E}-05$ & 2,34779E-08 \\
\hline & ९líquido & 6,91691E+04 & $-1,29476 \mathrm{E}+03$ & $9,89646 \mathrm{E}+00$ & $-3,77712 \mathrm{E}-02$ & $7,18670 \mathrm{E}-05$ & $-5,46027 \mathrm{E}-08$ \\
\hline & $\rho_{\text {vapor }}$ & $-6,94899 \mathrm{E}+04$ & $1,33501 \mathrm{E}+03$ & $-1,02185 \mathrm{E}+01$ & $3,89640 \mathrm{E}-02$ & $-7,40576 \mathrm{E}-05$ & 5,61974E-08 \\
\hline & ulíquido & $-5,69838 \mathrm{E}+06$ & $1,08348 \mathrm{E}+05$ & $-8,21431 \mathrm{E}+02$ & $3,13512 \mathrm{E}+00$ & $-5,96207 \mathrm{E}-03$ & 4,52879E-06 \\
\hline & $\mathrm{h}_{\text {líquido }}$ & $-6,50248 E+06$ & $1,23807 \mathrm{E}+05$ & $-9,39878 \mathrm{E}+02$ & $3,58746 \mathrm{E}+00$ & $-6,82396 \mathrm{E}-03$ & 5,18556E-06 \\
\hline & Slíquido & $-1,95430 \mathrm{E}+04$ & $3,64823 \mathrm{E}+02$ & $-2,71172 \mathrm{E}+00$ & 1,02447E-02 & $-1,93759 \mathrm{E}-05$ & 1,46596E-08 \\
\hline & cVlíquido & $-6,01566 \mathrm{E}+04$ & 1,16347E+03 & $-8,86488 \mathrm{E}+00$ & $3,36686 \mathrm{E}-02$ & $-6,36893 \mathrm{E}-05$ & 4,80283E-08 \\
\hline & cplíquido & $-3,00019 \mathrm{E}+06$ & $5,71849 \mathrm{E}+04$ & $-4,33731 \mathrm{E}+02$ & $1,63705 \mathrm{E}+00$ & $-3,07492 \mathrm{E}-03$ & 2,29986E-06 \\
\hline & SSlíquido & $2,19598 \mathrm{E}+04$ & $-3,80940 \mathrm{E}+02$ & $2,80510 \mathrm{E}+00$ & $-1,04591 \mathrm{E}-02$ & $1,95117 \mathrm{E}-05$ & $-1,45797 \mathrm{E}-08$ \\
\hline & $\mu_{\mathrm{JT}}$,liquido & $-3,51159 \mathrm{E}-03$ & $6,72054 \mathrm{E}-05$ & $-5,12284 \mathrm{E}-07$ & 1,94414E-09 & $-3,67388 \mathrm{E}-12$ & $2,76642 \mathrm{E}-15$ \\
\hline & $\mathrm{u}_{\mathrm{vapor}}$ & $8,95715 \mathrm{E}+06$ & $-1,67565 \mathrm{E}+05$ & $1,28539 \mathrm{E}+03$ & $-4,89877 \mathrm{E}+00$ & 9,31195E-03 & $-7,06908 \mathrm{E}-06$ \\
\hline & $\mathrm{h}_{\text {vapor }}$ & $1,05923 \mathrm{E}+07$ & $-1,98930 \mathrm{E}+05$ & $1,52627 \mathrm{E}+03$ & $-5,81732 \mathrm{E}+00$ & 1,10571E-02 & $-8,39472$ E-06 \\
\hline & S Sapor & $3,44993 \mathrm{E}+04$ & $-6,11716 \mathrm{E}+02$ & $4,57628 \mathrm{E}+00$ & $-1,71780 \mathrm{E}-02$ & $3,23005 \mathrm{E}-05$ & $-2,43198 \mathrm{E}-08$ \\
\hline & $\mathrm{cV}_{\text {vapor }}$ & $-5,55327 \mathrm{E}+04$ & 1,06940E+03 & $-8,14860 \mathrm{E}+00$ & 3,09917E-02 & $-5,87282 \mathrm{E}-05$ & 4,44069E-08 \\
\hline & $\mathrm{cp}_{\text {vapor }}$ & $-5,25437 \mathrm{E}+06$ & $1,00102 \mathrm{E}+05$ & $-7,59074 \mathrm{E}+02$ & $2,86436 \mathrm{E}+00$ & $-5,37896 \mathrm{E}-03$ & 4,02206E-06 \\
\hline & $\mathrm{SS}_{\text {vapor }}$ & $2,10300 \mathrm{E}+03$ & $-3,92657 \mathrm{E}+01$ & 3,07308E-01 & $-1,18535 \mathrm{E}-03$ & 2,27686E-06 & -1,76471E-09 \\
\hline & $\mu_{\mathrm{JT}, \mathrm{vapor}}$ & 9,75907E-03 & $-1,65300 \mathrm{E}-04$ & 1,13443E-06 & $-3,92026 \mathrm{E}-09$ & $6,80270 \mathrm{E}-12$ & $-4,73290 \mathrm{E}-15$ \\
\hline \multirow{18}{*}{$\mathrm{R} 718$} & plíquido & $-3,33096 \mathrm{E}+03$ & $6,32063 \mathrm{E}+01$ & $-4,83311 \mathrm{E}-01$ & $1,86286 \mathrm{E}-03$ & $-3,62194 \mathrm{E}-06$ & $2,84411 \mathrm{E}-09$ \\
\hline & $\mathrm{p}_{\text {vapor }}$ & $-3,33096 \mathrm{E}+03$ & $6,32063 \mathrm{E}+01$ & $-4,83311 \mathrm{E}-01$ & 1,86286E-03 & $-3,62194 \mathrm{E}-06$ & 2,84411E-09 \\
\hline & ९líquido & $-6,41831 \mathrm{E}+03$ & $1,07489 \mathrm{E}+02$ & $-6,26522 \mathrm{E}-01$ & $1,84235 \mathrm{E}-03$ & $-2,73530 \mathrm{E}-06$ & 1,63280E-09 \\
\hline & $\rho_{\text {vapor }}$ & $-6,57681 \mathrm{E}+00$ & 1,43204E-01 & $-1,23566 \mathrm{E}-03$ & 5,30481E-06 & $-1,13693 \mathrm{E}-08$ & $9,75792 \mathrm{E}-12$ \\
\hline & ulíquido & $-4,25081 \mathrm{E}+06$ & $5,14337 \mathrm{E}+04$ & $-2,87491 \mathrm{E}+02$ & 8,75692E-01 & $-1,33610 \mathrm{E}-03$ & 8,17394E-07 \\
\hline & $\mathrm{h}_{\text {líquido }}$ & $-4,25568 \mathrm{E}+06$ & $5,15231 \mathrm{E}+04$ & $-2,88155 \mathrm{E}+02$ & 8,78177E-01 & $-1,34080 \mathrm{E}-03$ & 8,20982E-07 \\
\hline & Slíquido & $-2,11747 \mathrm{E}+04$ & $2,52600 \mathrm{E}+02$ & $-1,34575 \mathrm{E}+00$ & 3,90692E-03 & $-5,81263 \mathrm{E}-06$ & 3,49833E-09 \\
\hline & cVlíquido & $-3,67541 \mathrm{E}+04$ & $6,05761 \mathrm{E}+02$ & $-3,58335 \mathrm{E}+00$ & 1,06936E-02 & $-1,62003 \mathrm{E}-05$ & 9,94026E-09 \\
\hline & cplíquido & $2,11557 \mathrm{E}+05$ & $-3,13688 \mathrm{E}+03$ & $1,89984 \mathrm{E}+01$ & $-5,75757 \mathrm{E}-02$ & 8,72833E-05 & $-5,29291 \mathrm{E}-08$ \\
\hline & SS $_{\text {líquido }}$ & $-2,60480 \mathrm{E}+04$ & $3,52189 \mathrm{E}+02$ & $-1,85487 \mathrm{E}+00$ & $5,03700 \mathrm{E}-03$ & $-7,01536 \mathrm{E}-06$ & 3,97375E-09 \\
\hline & $\mu_{\mathrm{JT} \text {, liquido }}$ & $-1,23430 \mathrm{E}-05$ & $1,76336 \mathrm{E}-07$ & $-1,04095 \mathrm{E}-09$ & $3,10362 \mathrm{E}-12$ & $-4,65769 \mathrm{E}-15$ & $2,81340 \mathrm{E}-18$ \\
\hline & $\mathrm{u}_{\mathrm{vapor}}$ & $1,86274 \mathrm{E}+06$ & $3,49180 \mathrm{E}+03$ & $-1,29560 \mathrm{E}+01$ & $3,80606 \mathrm{E}-02$ & $-5,06133 \mathrm{E}-05$ & $2,04686 \mathrm{E}-08$ \\
\hline & $\mathrm{h}_{\text {vapor }}$ & $1,83268 \mathrm{E}+06$ & 4,42114E+03 & $-1,57296 \mathrm{E}+01$ & 4,55308E-02 & $-5,84967 \mathrm{E}-05$ & 2,09833E-08 \\
\hline & S vapor & $4,92651 \mathrm{E}+04$ & $-4,19113 \mathrm{E}+02$ & $1,86029 \mathrm{E}+00$ & $-4,51432 \mathrm{E}-03$ & 5,80343E-06 & $-3,11154 \mathrm{E}-09$ \\
\hline & $\mathrm{cv}_{\text {vapor }}$ & $2,21362 \mathrm{E}+04$ & $-3,32330 \mathrm{E}+02$ & $2,10645 \mathrm{E}+00$ & $-6,60673 \mathrm{E}-03$ & 1,02551E-05 & $-6,27967 \mathrm{E}-09$ \\
\hline & $\mathrm{cp}_{\text {vapor }}$ & $2,43037 \mathrm{E}+04$ & $-3,58709 \mathrm{E}+02$ & $2,26523 \mathrm{E}+00$ & $-7,06660 \mathrm{E}-03$ & 1,08787E-05 & $-6,57073 \mathrm{E}-09$ \\
\hline & $\mathrm{SS}_{\text {vapor }}$ & $-4,99795 \mathrm{E}+02$ & 1,14691E+01 & $-6,54701 \mathrm{E}-02$ & 2,00603E-04 & $-3,06764 \mathrm{E}-07$ & 1,84264E-10 \\
\hline & $\mu_{\mathrm{JT}, \mathrm{vapor}}$ & 2,69095E-01 & $-3,69235 \mathrm{E}-03$ & 2,03979E-05 & $-5,66271 \mathrm{E}-08$ & 7,89231E-11 & $-4,41474 \mathrm{E}-14$ \\
\hline
\end{tabular}

